\title{
BMJ Open Exercise for patients with major depression: a systematic review with meta-analysis and trial sequential analysis
}

\author{
Jesper Krogh, ${ }^{1}$ Carsten Hjorthøj, ${ }^{1}$ Helene Speyer, ${ }^{1}$ Christian Gluud, ${ }^{2}$ \\ Merete Nordentoft ${ }^{1}$
}

To cite: Krogh J, Hjorthøj C, Speyer $\mathrm{H}$, et al. Exercise for patients with major depression: a systematic review with meta-analysis and trial sequential analysis. BMJ Open 2017;7:e014820. doi:10.1136/ bmjopen-2016-014820

- Prepublication history and additional material for this paper are available online. To view these files please visit the journal online (http://dx.doi. org/10.1136/bmjopen-2016014820).

Received 19 0ctober 2016 Revised 26 June 2017 Accepted 5 July 2017
CrossMark

${ }^{1}$ Faculty of Health Sciences, Mental Health Centre Copenhagen, University of Copenhagen, Copenhagen, Denmark

${ }^{2}$ Copenhagen Trial Unit, Centre for Clinical Intervention Research, Rigshospitalet, Copenhagen University Hospital, Copenhagen, Denmark

Correspondence to Dr Jesper Krogh; jesper.krogh@dadlnet.dk

\section{ABSTRACT}

Objectives To assess the benefits and harms of exercise in patients with depression.

Design Systematic review

Data sources Bibliographical databases were searched until 20 June 2017.

Eligibility criteria and outcomes Eligible trials were randomised clinical trials assessing the effect of exercise in participants diagnosed with depression. Primary outcomes were depression severity, lack of remission and serious adverse events (eg, suicide) assessed at the end of the intervention. Secondary outcomes were quality of life and adverse events such as injuries, as well as assessment of depression severity and lack of remission during follow-up after the intervention.

Results Thirty-five trials enrolling 2498 participants were included. The effect of exercise versus control on depression severity was -0.66 standardised mean difference (SMD) $(95 \% \mathrm{Cl}-0.86$ to $-0.46 ; \mathrm{p}<0.001$; grading of recommendations assessment, development and evaluation (GRADE): very low quality). Restricting this analysis to the four trials that seemed less affected of bias, the effect vanished into -0.11 SMD $(-0.41$ to $0.18 ; p=0.45$; GRADE: Iow quality). Exercise decreased the relative risk of no remission to 0.78 ( 0.68 to 0.90 ; $\mathrm{p}<0.001$; GRADE: very low quality). Restricting this analysis to the two trials that seemed less affected of bias, the effect vanished into 0.95 (0.74 to 1.23 ; $p=0.78)$. Trial sequential analysis excluded random error when all trials were analysed, but not if focusing on trials less affected of bias. Subgroup analyses found that trial size and intervention duration were inversely associated with effect size for both depression severity and lack of remission. There was no significant effect of exercise on secondary outcomes.

Conclusions Trials with less risk of bias suggested no antidepressant effects of exercise and there were no significant effects of exercise on quality of life, depression severity or lack of remission during followup. Data for serious adverse events and adverse events were scarce not allowing conclusions for these outcomes.

Systematic review registration The protocol was published in the journal Systematic Reviews: 2015; 4:40.
Strengths and limitations of this study

- The protocol for this review has previously been published.

- Using meta-regression analysis, trial sequential analysis and the grading of recommendations assessment, development and evaluation system, the conclusions from this review is based on a firm and transparent platform.

- Based on an extensive literature search, this review included 35 trials allocating almost 2500 participants diagnosed with depression to exercise or control interventions than could be analysed.

- The effect estimates are largely based on trials at high risk of bias.

- Effect estimates from included trials had considerable heterogeneity.

\section{INTRODUCTION}

Depression is a common disorder affecting up to $17 \%$ of the population during their lifetime. ${ }^{12}$ Based on data from WHO, depression is ranked as the second largest healthcare problem globally, in terms of years lived with disability. ${ }^{3}$ Depending on its severity, depression is often treated using psychotherapy, antidepressants or a combination of both. However, the clinical benefits of antidepressants $^{4-6}$ and psychotherapy ${ }^{7-9}$ has been challenged. Both treatments are costly in terms of time and money and may also have adverse effects. Compliance with antidepressant treatment is poor; the dropout rate in clinical trials is reported to be between $12 \%$ and $40 \%$ within the initial 6-8 weeks of treatment. ${ }^{410}$

The weakness of evidence for the beneficial effect of current interventions, along with problems related to low compliance and harms, has resulted in an interest in using alternative interventions. The use of exercise as an intervention has attracted considerable attention, and various forms 
of exercise varying in intensity have been assessed in a number of randomised clinical trials to test their effectiveness as a treatment for patients with depression. In 2011, we published a meta-analysis of randomised clinical trials examining the effect of exercise on depressive symptoms in patients with clinical depression. ${ }^{11}$ The results suggested that referring patients with clinical depression to exercise programme was associated with a small-to-moderate effect on depressive symptoms. However, restricting the analysis to three trials at low risk of bias, the effect estimate was non-significant. Since 2011, other reviews have been published on the effect of exercise on depressive symptoms, ${ }^{12}$ in older people, ${ }^{13}$ and in patients with chronic illnesses. ${ }^{14}$ However, none of these reviews addressed the specific population of adults diagnosed with major depression according to valid diagnostic criteria, such as the International Classification of Diseases ${ }^{15}$ or the Diagnostic and Statistical Manual of Mental Disorders. ${ }^{16}$ The reviews contained a number of trials that included volunteers who were defined as being depressed on the basis of psychometric testing (eg, Beck Depression Inventory ${ }^{17}$ ), as opposed to individuals with a clinical diagnosis of major depression. Furthermore, several randomised clinical trials investigating the effect of exercise in clinically depressed individuals have been published since our 2011 review. $^{11}$

The objectives of the present systematic review are to investigate the beneficial and harmful effects of exercise, in terms of severity of depression, lack of remission, quality of life and suicide versus controls with or without co-interventions in adults with a clinical diagnosis of major depression. The current systematic review differs from our previous review in a number of aspects. ${ }^{11}$ We only considered trials including participants diagnosed with depression according to a validated diagnostic system. We also included trials including participants with somatic comorbidity, for example, cancer or diabetes. The harmful effects of exercise interventions are also addressed, the intervention effects being assessed according to the grading of recommendations assessment, development and evaluation (GRADE) framework, and bibliographical searches have been extended to include a Chinese and a South American database until 2016.

\section{METHODS/DESIGN}

The protocol for this review has previously been published. ${ }^{18}$

\section{Search strategy}

The following bibliographical databases was searched: CENTRAL, MEDLINE, EMBASE, Science Citation Index (Web of Science), LILACS and Wanfang using medical subject headings (MeSH or similar) when possible or text word terms: depression, depressive disorder and exercise, aerobic, non-aerobic, physical activity, physical fitness, walking, jogging, running, bicycling, swimming, strength or resistance (see online supplementary material S1 for an example of a bibliographical search). The main search was conducted in August 2015, and the latest search was conducted on 20 June 2017.

\section{Trial selection}

One investigator (JK) examined titles and abstracts to remove obviously irrelevant reports. Two investigators (JK+HS) examined full-text reports and abstracts determining compliance with inclusion criteria. A trial was considered eligible if it was a randomised clinical trial including participants diagnosed as having major depression according to a valid and recognised diagnostic system (ie, Research Diagnostic Criteria, ${ }^{19}$ International Classification of Diseases (ICD) ${ }^{15}$ or Diagnostic and Statistical Manual of Mental disorders (DSM) ${ }^{16}$ and included participants aged $>17$ years. Abstracts and full-text reports were included.

Trials were excluded if they measured depression immediately after a single bout of exercise, compared one form of exercise versus another, or compared different exercise intensities without including a control group. The trials had to allocate participants to an exercise intervention versus a control group (ie, exercise vs a control group receiving no intervention or treatment as usual or an attention control using light exercise) or using exercise as an add-on treatment (ie, exercise plus usual treatment in the experimental group vs usual treatment alone in the control group). Exercise intervention was defined as a systematic physical intervention with the intention to increase muscle strength and/or cardiovascular fitness, for example, running, swimming or weight lifting. In case of attention control, it should specifically be mentioned by the authors of the trial report that the intervention was intended as a control intervention.

\section{Outcomes}

The primary outcomes were: 1) depressive symptoms measured on a continuous scale assessed at the end of the intervention; 2) lack of remission, that is, a binary outcome of the proportion of participants in each intervention group of the trial who did not obtain remission at the end of the intervention according to the authors' own definition and 3) serious adverse events defined according to International Council for Harmonisation, Good Clinical Practice (ICH-GCP) as any untoward medical occurrence that was life threatening, resulted in death or persistent or significant disability (ICH-GCP 1997). ${ }^{20}$ Serious adverse events accordingly include suicide attempts as well as suicides. The secondary outcomes were quality of life, non-serious adverse events (eg, muscle injuries) as well as depressive symptoms and lack of remission assessed after the intervention.

\section{Data extraction}

Two authors (JK, HS) independently extracted data using a prepiloted structured form. Any discrepancies in the data extraction or inclusion/exclusion of trials was resolved by referring to the original papers. CG or MN 
assisted as adjudicator in cases of disagreements. Data extraction included, in addition to outcomes, information regarding country of origin, number of randomised participants, number of participants included in efficacy analysis, mean age of participants, diagnostic system, baseline assessment of depression severity, type of intervention, frequency of intervention and duration of intervention. Continuous outcomes were preferred in the following order: postintervention scores with corresponding SD, mean change from baseline with SD, mean difference between groups postintervention and reported outcomes were preferred to figures. JK and $\mathrm{CH}$ independently performed the assessment of bias domains. The authors JK, CG and MN have previously published trial reports assessing the effect of exercise in participants with depression, ${ }^{21} 22$ and to reduce the risk of academic bias two additional authors were included in the current systematic review (CH, HS).

\section{Risk of bias assessment}

Definitions in the assessment of bias risk of a trial was conducted according to the Cochrane Handbook for Systematic Reviews of Interventions ${ }^{23}$ of the following domains: allocation sequence generation, allocation concealment, blinding of participants and personnel, blinding of outcome assessors, incomplete outcome data, selective outcome reporting, for-profit bias and other bias. Trials assessed as having 'low risk of bias' in all of the above specified domains were considered 'trials at low risk of bias'. Trials assessed as having 'uncertain risk of bias' or 'high risk of bias' in one or more of the above specified domains were considered trials at 'high risk of bias'. In line with our previous systematic review ${ }^{11}$ and the latest Cochrane review on exercise for depression, ${ }^{24}$ trials at low risk of bias in the allocation concealment domain, blinded outcome assessment domain and the incomplete outcome data domain were characterised as "trials potentially having less risk of bias than other trials at high risk of bias'. Trials assessing the effect of behavioural interventions are rarely able to mask the allocation, and participants and healthcare providers are therefore not blinded. Therefore, we will also report the number of trials at low risk of bias in the remaining domains.

\section{Data synthesis and analysis}

In order to be able to include all of the trials in our meta-analysis, estimates of standardised mean difference (SMD) for each individual trial was carried out. SMD is the mean difference in depression score between the exercise and control groups divided by the pooled SD at follow-up. The result is a unit-free effect size. By convention, SMD effect sizes of $0.2,0.5$ and 0.8 are considered small, medium and large intervention effects. ${ }^{23}$ For dichotomous variables, we calculated the risk ratio (RR) with a $95 \%$ CI. It was expected that some trials would have several intervention groups. Data from the experimental groups were pooled and compared with the data from the control group. In case of discrepancies between the random-effects model analysis and the fixed-effect model analysis, both results are reported; otherwise, only results from the random-effects analysis are reported. The degree of heterogeneity was quantified using the $\mathrm{I}^{2}$ statistic, ${ }^{25}$ which can be interpreted as the percentage of variation observed between the trials attributable to between-trial differences, rather than sampling error (chance). Heterogeneity was explored by analyses of subgroups (see below).

For the primary outcomes, trial sequential analysis was performed. ${ }^{26}{ }^{27}$ In order to calculate the required information size and the cumulative Z-curve's eventual breach of relevant trial sequential monitoring boundaries, the required information size for the primary continuous outcome was based on type I error of $5 \%$, a beta of $10 \%$, the $\mathrm{SE}$ of the meta-analysis and a minimal difference of three points on the Hamilton Depression Scale, 17 items $\left(\right.$ HAM-D $\left.{ }_{17}\right){ }^{18}$ Post hoc we calculated the required information size including all trials. This was done by converting effect estimates from trials reporting other outcome scales into the HAM-D ${ }_{17}$ scale as described by Thorlund $e t a l .{ }^{28}$ In order to calculate the required information size and the cumulative Z-curve's eventual breach of relevant trial sequential monitoring boundaries, the required information size for lack of remission was based on type I error of $5 \%$, a beta of $10 \%$, the proportion of participants in the control group with the outcome and a relative risk reduction of $15 \%$ and $30 \%$.

Bayes factors were calculated for all primary outcomes. ${ }^{29}$ Low $p$ values suggest that we can reject the null-hypothesis. But even a low p value from a meta-analysis can be misleading if there is also a low probability that data are compatible with the anticipated intervention effect. In other words, the probability that the actual measured difference in effect of the compared interventions resulted from an a priori anticipated 'true' difference needs to be considered. For this purpose, it is helpful to calculate the Bayes factor, which is the ratio of the $\mathrm{p}$ value probabilities of the meta-analysis result divided by the probability of the anticipated effect, or 'true' effect. ${ }^{29}$ As suggested by Jakobsen et $a l,{ }^{29}$ a Bayes factor $<0.1$ together with a low $p$ value suggest, if bias can be ruled out, that the observed result is compatible with the a priori expected effect. If the Bayes factor is $>0.1$, the result is not compatible with the a priori expected effect and the effect may be lower.

To assess the potential impact of missing data (incomplete outcome data bias), we did sensitivity analysis of missing data using the following strategy: a 'best-worst' case scenario was assessed, assuming that all participants lost to follow-up in the intervention group had a beneficial outcome (the group mean minus $1 \mathrm{SD}$ ), and all those with missing outcomes in the control group have had a harmful outcome (the group mean plus 1 SD and $2 \mathrm{SD})$. In addition, the reverse 'worst-best-case' scenario analysis was also performed. ${ }^{29}$ Missing data for the 'lack of remission' outcome were imputed in sensitivity analysis according to the following scenarios ${ }^{30}: 1$ ) poor outcome analysis: assuming that all of the drop-outs/participants 
lost from both the experimental and the control arms experienced the outcome, including all randomised participants in the denominator; 2) good outcome analysis: assuming that none of the drop-outs / participants lost from the experimental and the control arms experienced the outcome, including all randomised participants in the denominator; 3) extreme case analysis favouring the experimental intervention ('best-worse' case scenario): none of the drop-outs/participants lost from the experimental arm, but all of the drop-outs/participants lost from the control arm experienced the outcome, including all randomised participants in the denominator and 4) extreme case analysis favouring the control ('worst-best' case scenario): all of the drop-outs/participants lost from the experimental arm, but none from the control arm experienced the outcome, including all randomised participants in the denominator.

\section{Subgroup analyses}

In subgroup analyses, the possible effects of variables on intervention effects on outcomes and heterogeneity were compared. Trials potentially having less risk of bias (ie, trials with adequate allocation concealment, blinded outcome assessment and intention-to-treat analysis) were compared with trials at high risk of bias. The effect of age was assessed by comparing trials including older participants (mean age $>59$ years) to trials including younger participants (mean age $<60$ years). The effect of type of exercise was assessed by comparing trials using group exercises compared with trials using individual exercise. The effect of duration of intervention was assessed by comparing trials with short duration of intervention to trials with long duration of intervention splitting by the median time of duration. The effect of type of control group was assessed by comparing trials using attention control to trials with waitlist controls and comparing trials with exercise as add-on to medication to trials not using any medication. In addition, a within-study comparison of low-dose exercise versus high-dose exercise in trials using different exercise intensities was performed. The effect of comorbid somatic disease was assessed by comparing the effect estimates from trials including participants with depression compared with trials including participants with depression in addition to a somatic disease. Publication bias was assessed by visual inspection of a funnel plot and by Egger's test and if publication bias plausible Duval's and Tweedie's trim and fill procedure was conducted. $^{31}$

We assessed and graded the evidence according to the GRADE for high risk of bias, imprecision, indirectness, heterogeneity and publication bias. ${ }^{32}$ Based on this assessment, the intervention was graded accordingly: 'high quality'-we are very confident that the true effect lies close to that of the estimate of the effect; 'moderate quality' -we are moderately confident in the effect estimate. The true effect is likely to be close to the estimate of the effect, but there is a possibility that it is substantially different; 'low quality'-our confidence in the effect estimate is limited: the true effect may be substantially different from the estimate of the effect; "very low quality'-we have very little confidence in the effect estimate: the true effect is likely to be substantially different from the estimate of the effect. ${ }^{33}$

\section{Deviations from our protocol}

Post hoc we included trials using the Chinese Classification of Mental Disorders (CCMD) as well as a few trials including participants classified as having 'minor depression'. The CCMD system closely adhere to the ICD and DSM systems and have been found highly compatible in field studies, so these studies were included. ${ }^{34} \mathrm{~A}$ few trials included some participants classified as having 'minor depression' according to the trials chosen diagnostic system (eg, DSM), and it is questionable if these participants have major depression. We therefore decided to include these trials and to conduct a subgroup analysis exclusively including participants with major depression. To further explore heterogeneity, we post hoc included subgroup analysis comparing intervention effects in inpatients and outpatients as well as an analysis according to trial size. Trials were divided into small or large trials using the median of total $\mathrm{n}$ included in the efficacy analysis. The effect of exercise capacity was post hoc assessed by comparing trials with a high increase in maximal oxygen uptake $\left(\mathrm{VO}_{2} \mathrm{max}\right)$ with studies with lower increase in maximal oxygen uptake. Assessment of exercise capacity was based on the increase of $\mathrm{VO}_{2} \max$ in the intervention groups and trials were stratified to either high or low increase in exercise capacity by median. We did not conduct trial sequential analysis based on a relative risk reduction of $30 \%$ of lack of remission as this was an implausible effect.

\section{Participant involvement \\ Depressed participants were not involved in this study.}

\section{RESULTS}

\section{Bibliographical search and trial characteristics}

The main bibliographical search was conducted on 26 August 2015 and the final updates were conducted on 20 June 2017. As illustrated in online supplementary figure S1, we identified 45 publications reporting the effect of exercise on depressive symptoms in 35 randomised clinical trials. ${ }^{21} 2235-78$ Seventeen trials were conducted in Europe, ${ }^{21} 2240495253556165-687475777980$ eight in the USA, 3839434560647681 six in Asia, ${ }^{4769-73}$ two in Australia ${ }^{54} 58$ and two in South America. ${ }^{56} 63$ A total of 2630 participants were randomised and 2498 were included in the efficacy analysis of benefit. Ten trials included inpatients ${ }^{47} 49566769-7379$ and five trials included participants with a mean age $>60$ years. ${ }^{5254586061}$ No trials exclusively included participants with comorbid somatic disease. Four trials reported the continuous outcome as mean change from baseline in each group with a corresponding $\mathrm{SD},{ }^{39} 536568$ and one trial presented data as 
mean difference between groups postintervention. ${ }^{40}$ The remaining trials reported postscores in each group with corresponding SD (see table 1 for trial characteristics).

\section{Bias risk assessment}

Sequence generation was adequate in 15/35 (43\%), allocation concealment was adequate in 13/35 (37\%) trials, blinding of participants and trial personnel was adequate in $0 / 35(0 \%)$, blinded outcome assessment was performed in 16/35 (46\%), low risk of bias in the 'incomplete outcome data' domain was found in 12/35 (34\%) trials, selective outcome reporting domain was adequate in $31 / 35(89 \%)$, for-profit bias domain was adequate in $19 / 35(54 \%)$ and $25 / 35(71 \%)$ were free of other bias. Accordingly, all trials were at high risk of bias. Given the nature of the intervention, no trial had blinded participants or trial personnel, however, two trials had low risk of bias in all other bias domains. ${ }^{22}{ }^{54}$ Five trials $(16 \%)$ were sponsored by for-profit organisations: three trials were supported by pharmaceutical companies, ${ }^{53} 7982$ one trial by a company producing fitness machines ${ }^{45}$ and one trial by an insurance company. ${ }^{21}$ According to our a priori defined criteria, 4/35 (11\%) trials potentially had less risk of bias than the other trials at high risk of bias ${ }^{21} 225456$ (see table 2 for details on assessment of risk of bias).

\section{Primary outcomes}

The effect of exercise on depression severity

All included trials provided a continuous outcome on depression severity for the assessment of the exercise intervention encompassing 2498/2630 randomised participants $(95 \%)$. The effect of intervention versus control was a SMD of -0.66 (95\% CI -0.86 to -0.46 ; $\mathrm{p}<0.001$ ) (figure 1). This corresponds to an effect on the HAM-D $_{17}$ scale of -4.1 (95\% CI -5.3 to -2.9$)$ points.

\section{Missing data}

Missing outcome analysis for depression as a continuous outcome did not markedly change the effect estimates. The least favourable outcome for the exercise intervention was the worse/best outcome analysis using $+2 \mathrm{SD}$ resulting in an effect estimate of -0.57 SMD (95\% CI -0.78 to -0.36 ; $\mathrm{p}<0.001$ ) (see online supplementary table $\mathrm{S} 1$ ).

\section{Heterogeneity and subgroup analysis}

The $\mathrm{I}^{2}$ was $81 \%$ suggesting substantial heterogeneity. Subgroup analysis revealed that the effect estimates for trials potentially having less risk of bias was -0.11 SMD ( $95 \%$ CI -0.41 to $0.18 ; p=0.45 ; I^{2}=62 \%$ ) compared with that of the trials at high risk of bias -0.75 SMD $(-0.98$ to $-0.52 ; \mathrm{p}<0.001 ; \mathrm{I}^{2}=81 \%$ ) (test of subgroup difference, $\mathrm{p}<0.001)$. In addition, trials including 50 participants or less had a pooled estimate of -1.11 ( -1.52 to -0.72 ; $\left.\mathrm{p}<0.001 ; \mathrm{I}^{2}=78 \%\right)$ compared with that of larger trials of $-0.37\left(-0.57\right.$ to $\left.-0.18 ; \mathrm{p}<0.001 ; \mathrm{I}^{2}=75 \%\right)$ (test of subgroup difference, $p=0.001$ ). Trials of short duration of intervention $(<10$ weeks $)$ had an SMD of $-0.92(-1.09$ to -0.74 ; $\left.\mathrm{p}<0.001 ; \mathrm{I}^{2}=14 \%\right)$ compared with trials with longer duration of intervention, -0.49 ( -0.75 to -0.23 ; $\mathrm{p}<0.001$;
$\mathrm{I}^{2}=83 \%$ ) (test of subgroup difference, $\mathrm{p}=0.007$ ). Effect estimates from trials including participants with minor depression compared with trials exclusively including participants with major depression did not differ (test of subgroup difference, $p=0.53$ ).

Four trials allocated 206 participants to different exercise intensities/doses. ${ }^{45} 587383$ Comparing the postintervention depression scores for participants allocated to either high-intensity/high-dose versus low-intensity/ low-dose exercise showed a difference of -0.40 SMD $\left(95 \%\right.$ CI -0.67 to $\left.-0.12 ; \mathrm{p}=0.005 ; \mathrm{I}^{2}=0 \%\right)$ in favour of high-intensity/high-dose exercise. As shown in table 3, no other trial characteristic significantly explained any of the observed heterogeneity (see online supplementary table S2 for trial characteristics used to explore heterogeneity.

Trial sequential analysis and diversity adjusted required information size

The diversity adjusted required information size for HAM-D ${ }_{17}$ as a continuous outcome was calculated based on our anticipated intervention effect of a minimal relevant difference of 3.0 HDRS points, an SD of 6.78 points, a risk of type I error of 0.05 , a power of $90 \%$ and the observed diversity of $92 \%$ to 2610 participants. Only 14 trials reported results from HAM-D ${ }_{17}^{21} 22383943445253555658687083$ with an accrued 1124 participants. As shown in online supplementary figure S2, the cumulative Z-curve just crossed the trial sequential monitoring boundary for benefit. With the aforementioned settings, the pooled estimate is therefore less likely to be a random finding due to lack of power or multiple testing if bias could be ignored. Post hoc, we calculated the adjusted required information size for HAM-D ${ }_{17}$ including all trials as shown in online supplementary figure S3. As with the original analysis, the Z-curve crossed the trial sequential monitoring boundary for benefit supporting that the pooled estimate is less likely to represent a type 1 error if bias could be ignored.

\section{Bayes factor}

Fourteen trials reported effect estimates using the HAM-D ${ }_{17}{ }^{21} 22383943455253556368708384$ Based on these trials, Bayes factor was calculated $\left(\delta=-3.37 ; \mathrm{SE}_{\delta}=0.96\right.$; $\left.\mu_{\mathrm{a}}=-3.0\right)$ and was found to be 0.002 , which is below the Bayes factor threshold for significance of 0.1 , supporting the intervention effect if bias could be ignored.

\section{Publication bias}

Inspection of the funnel plot (not shown) suggested that small trials with small or no effect of exercise were missing (see online supplementary figure S4). Egger's test supported the suspicion of publication bias, $\mathrm{p}<0.00001$. Using the Duval's and Tweedie's trim and fill procedure, the estimate was reduced into -0.27 SMD $(95 \%$ CI -0.50 to -0.05$)$. This corresponds to an effect on the HAM-D ${ }_{17}$ scale of -1.7 (95\% CI -3.1 to -0.31$)$ points.

The effect of exercise on depression-lack of remission

Nineteen trials, randomising 1825 participants and including 1639 participants $(90 \%)$ in final analysis 


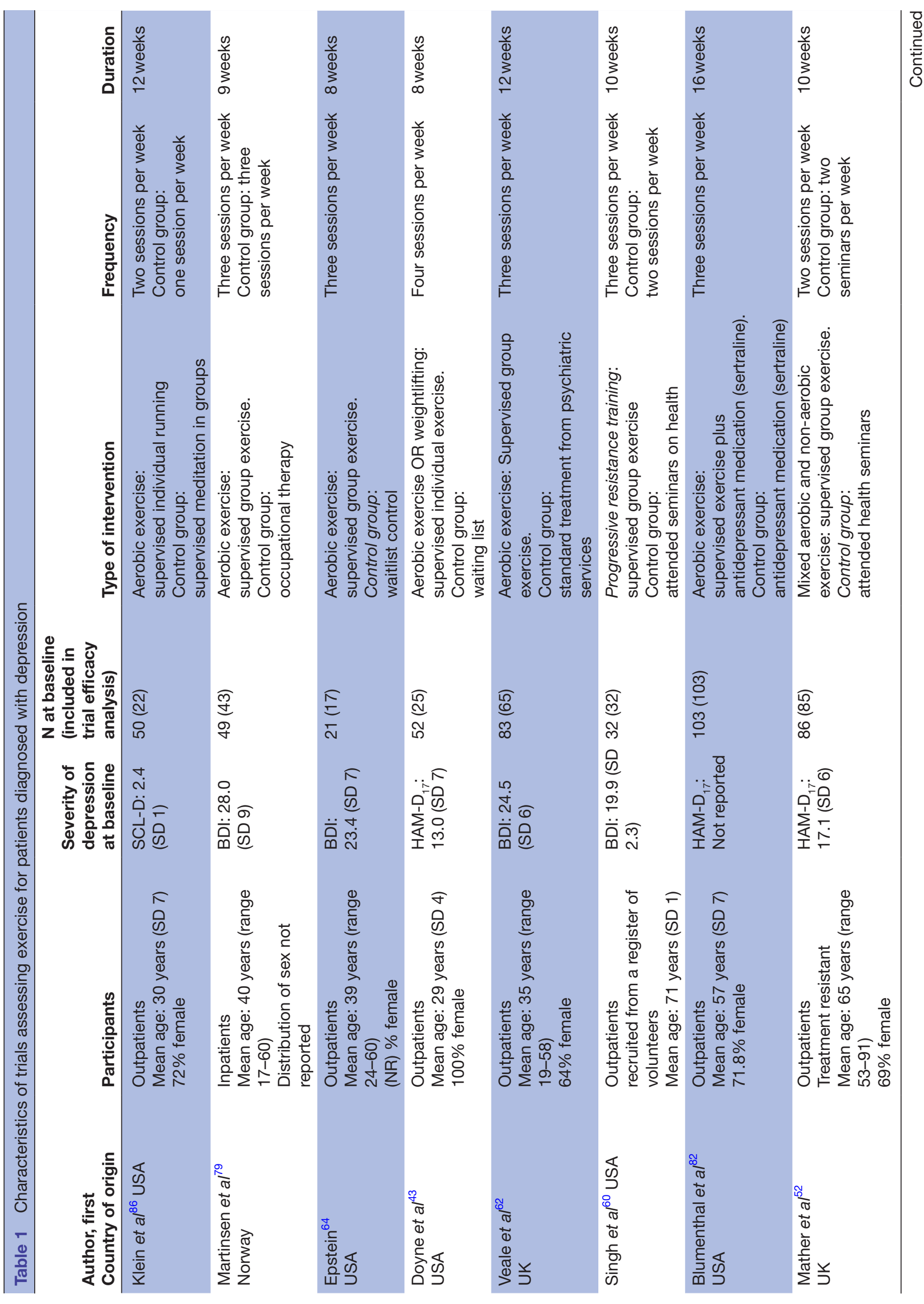

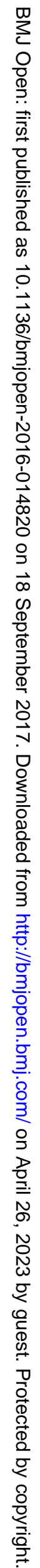




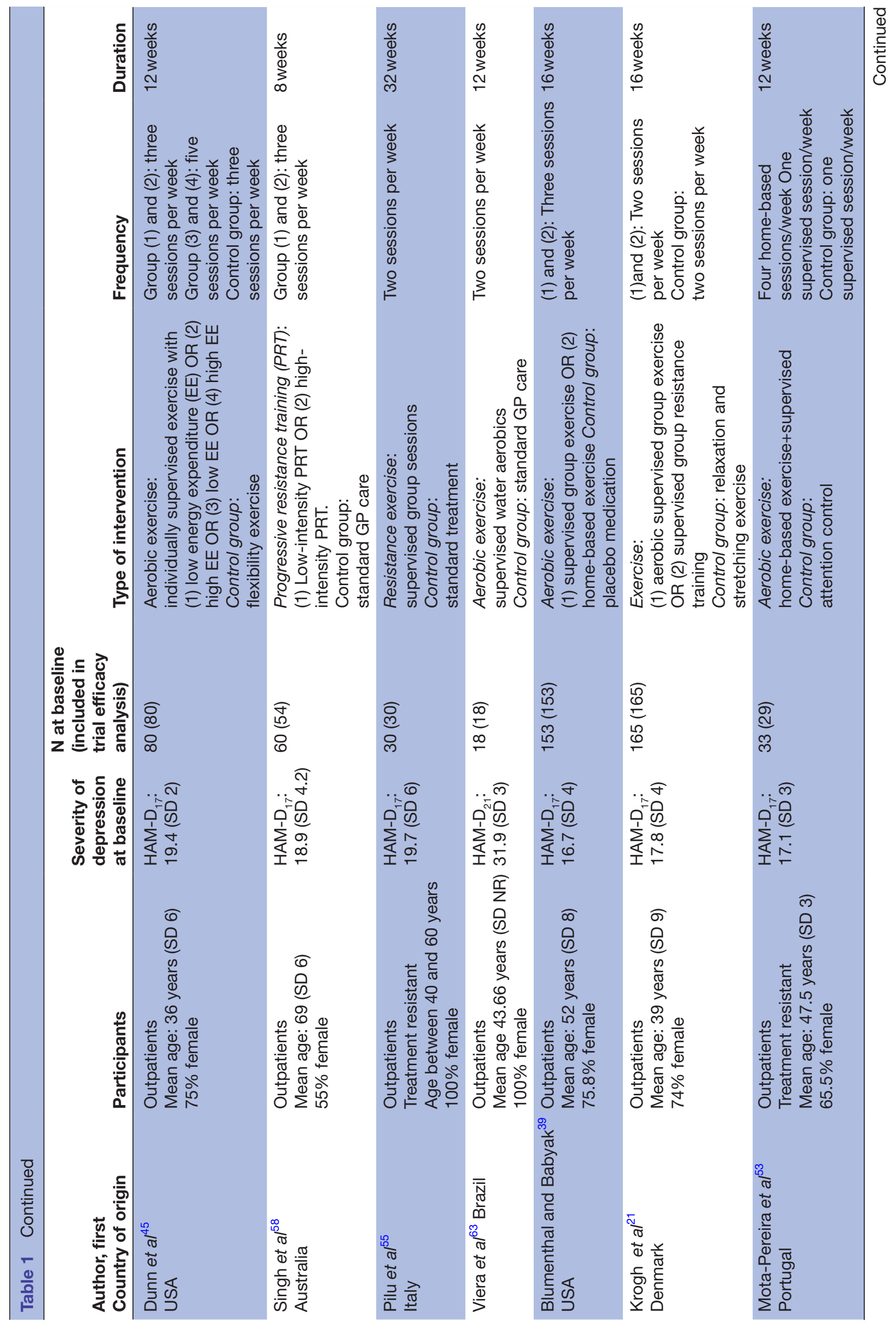




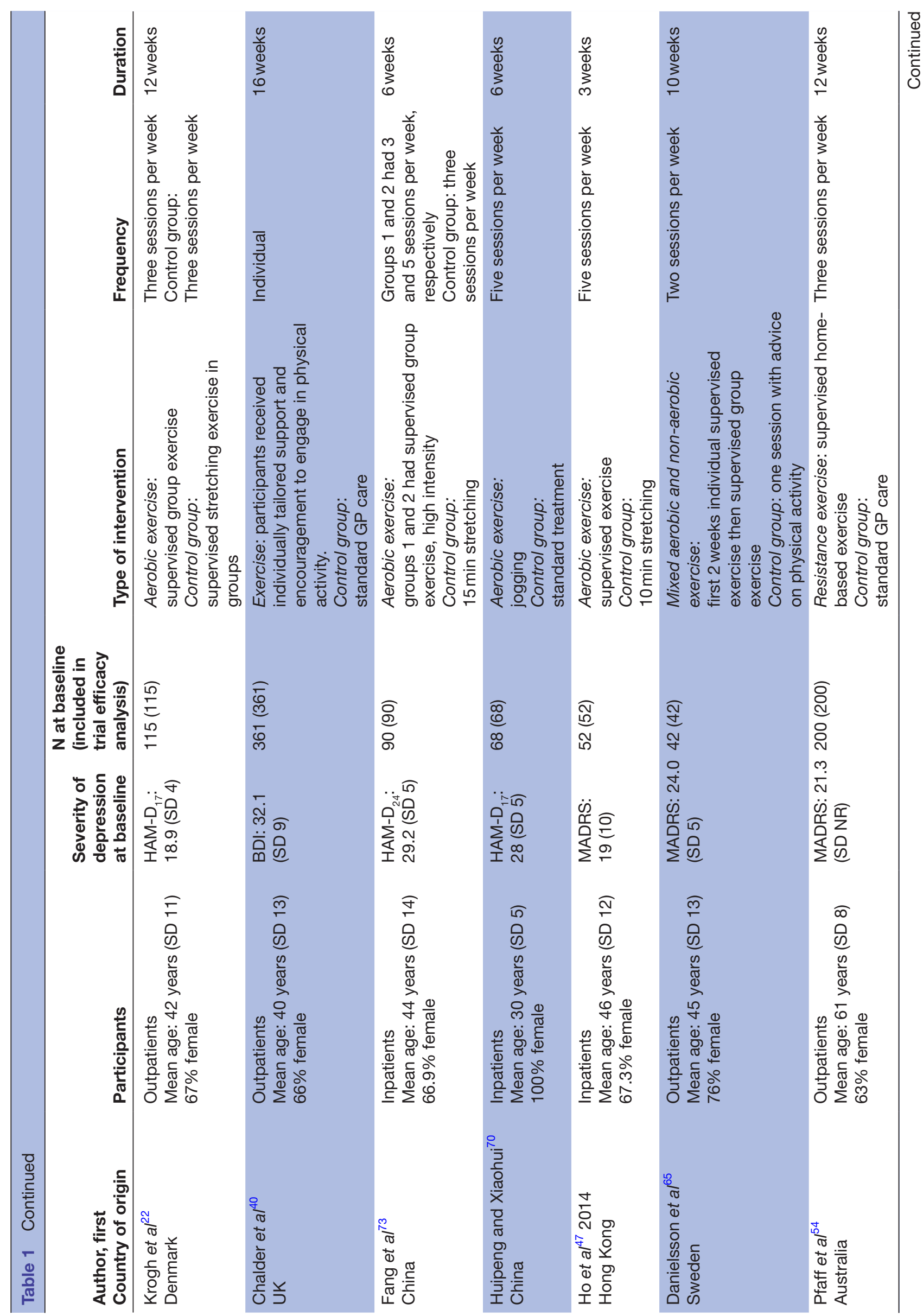




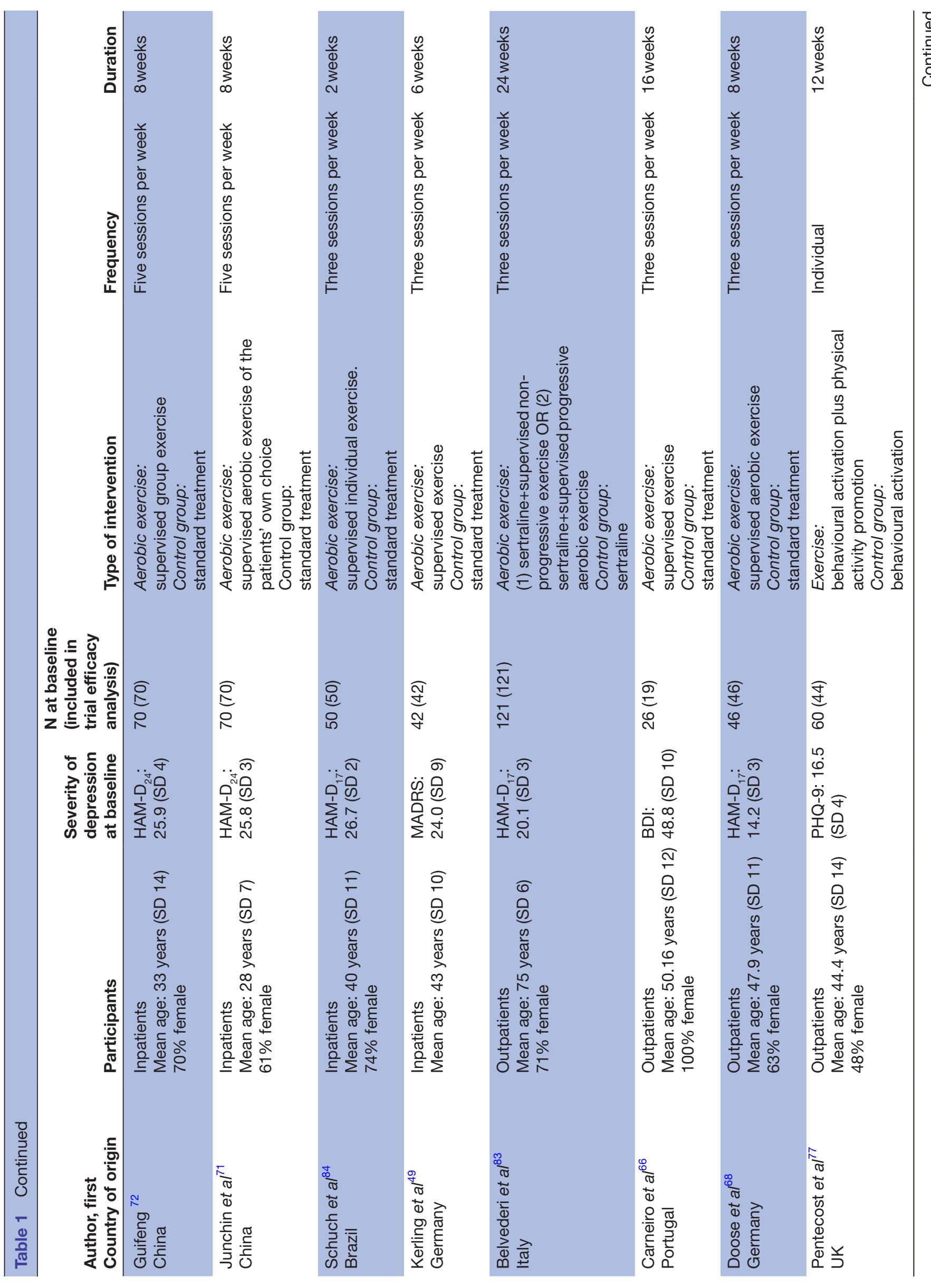




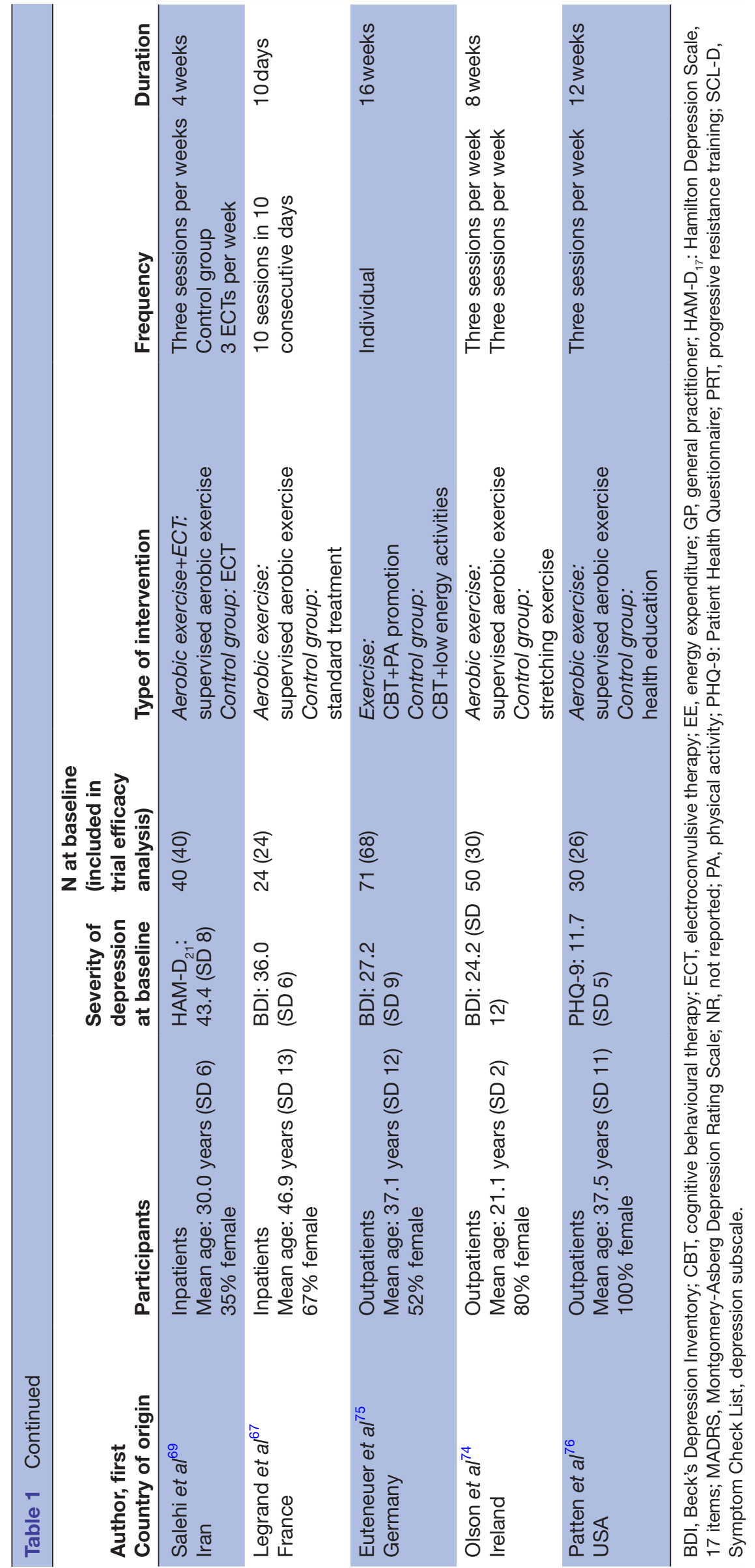




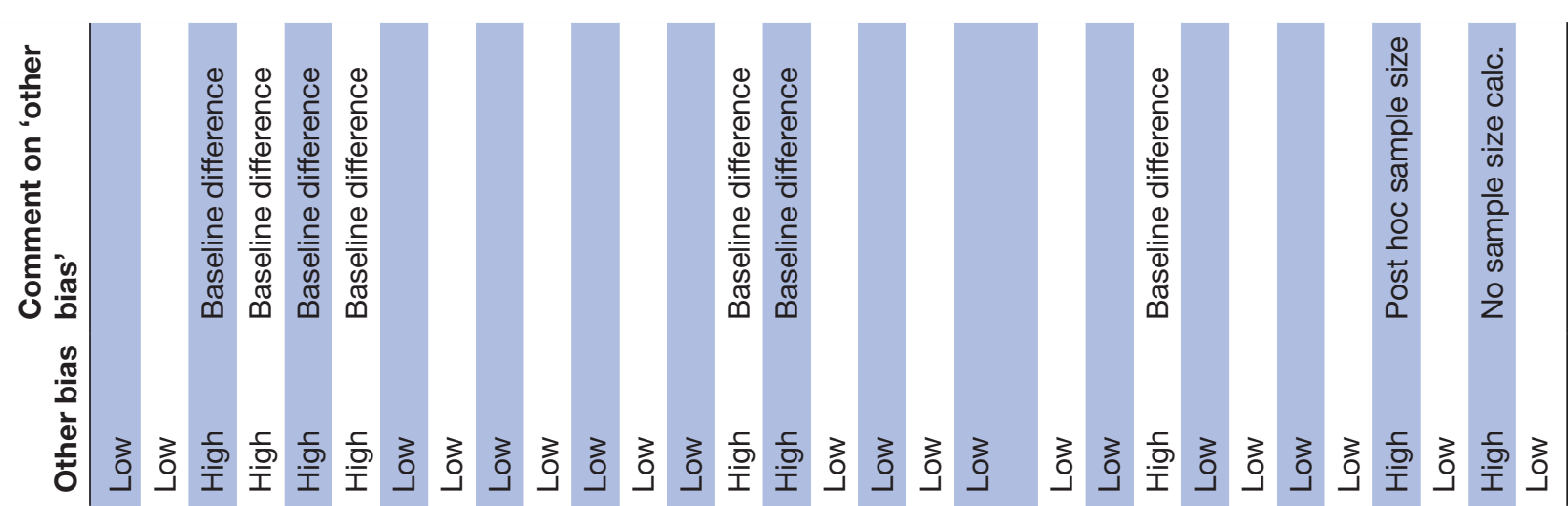

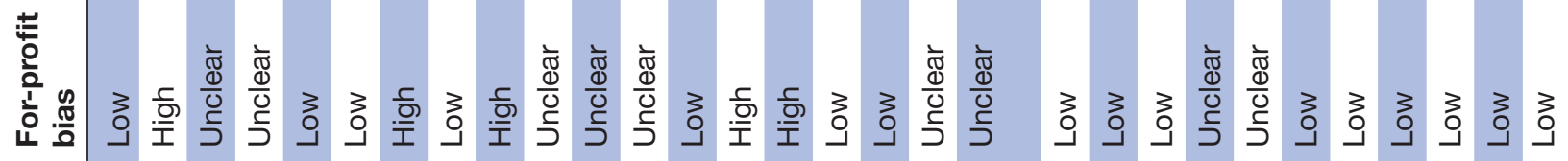

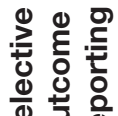

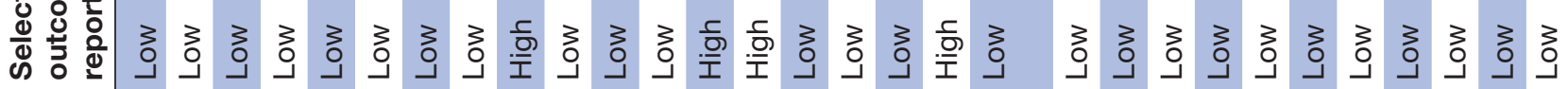

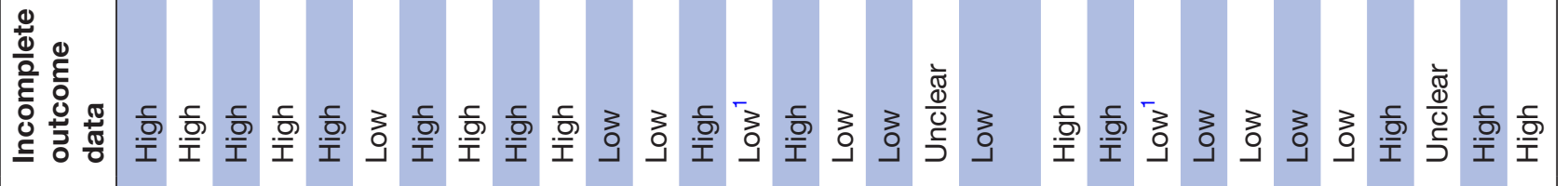
¿

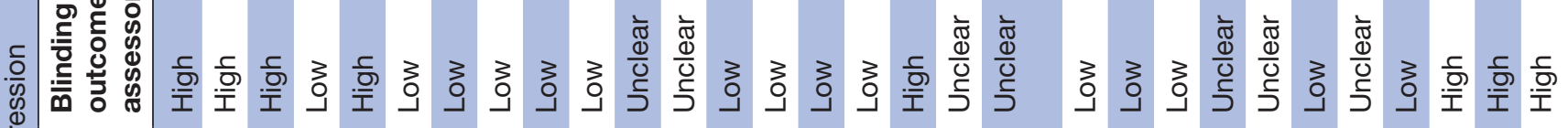
क्ष

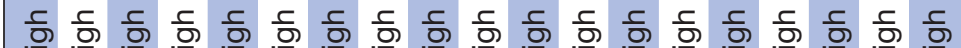

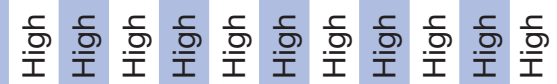

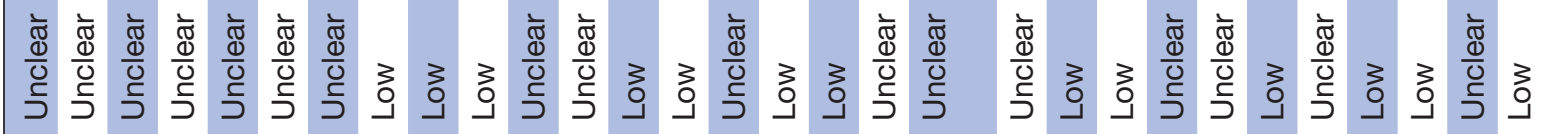

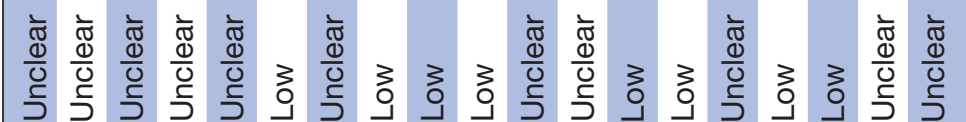

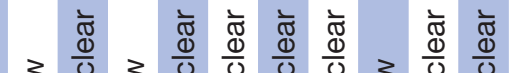 3.}

$\subseteq$

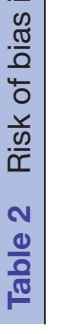

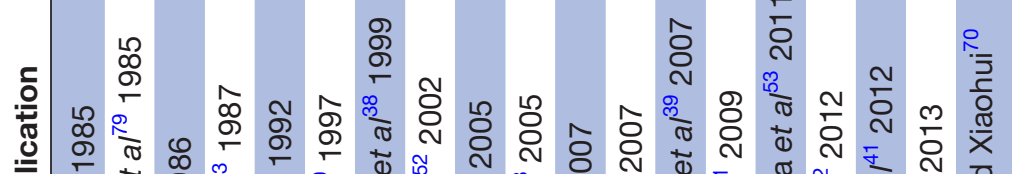

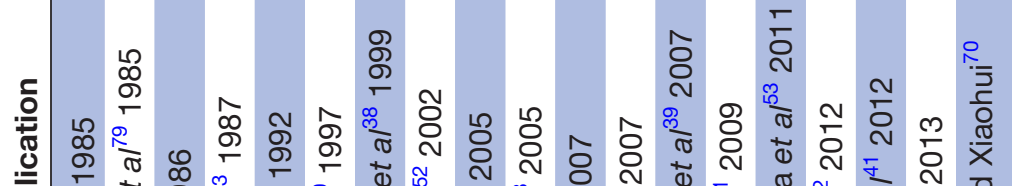

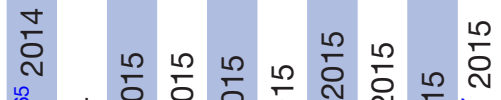
oำ

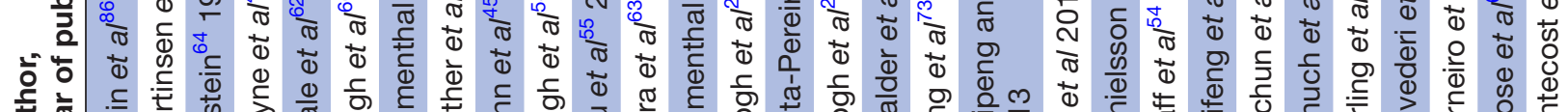

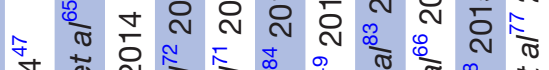

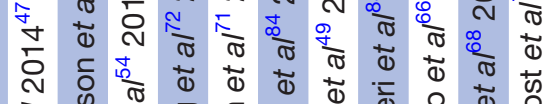

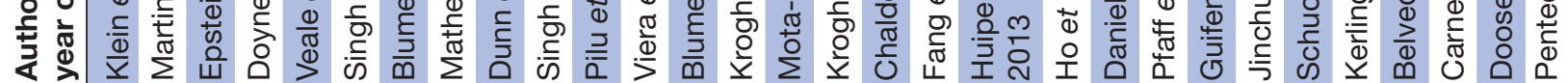




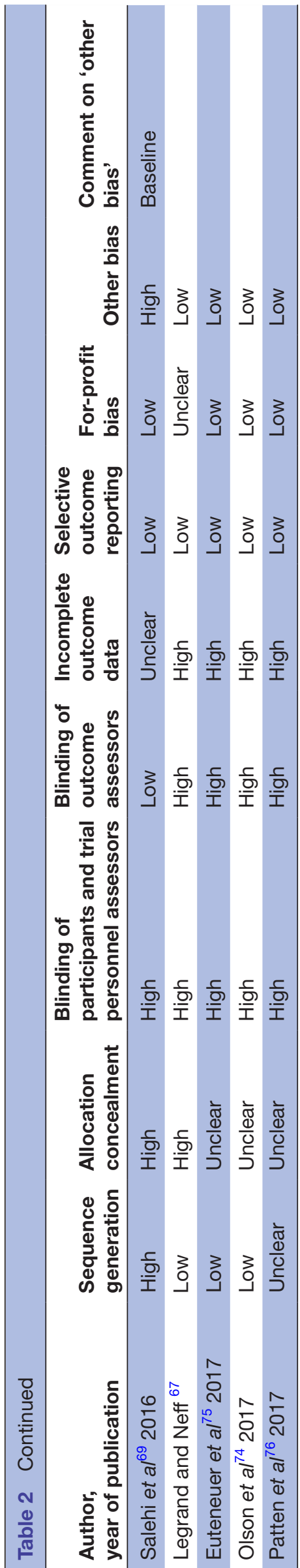

reported remission as an outcome. ${ }^{21} 2238-40434547495354$ $5660616568-7072$ Remission postintervention was defined in various ways: a postintervention score on the HAM-D $\mathrm{D}_{17}<8$ points, ${ }^{445356970}$ not fulfilling the DSM criteria for depression and $\mathrm{HAM}-\mathrm{D}_{17}<8$ points, ${ }^{21} 2239$ not fulfilling the DSM criteria for depression, ${ }^{385460}$ a BDI score $<9$ points, ${ }^{43}$ a BDI score $<10$ points, ${ }^{40}$ a HAM-D ${ }_{17}$ score $<10$ points, ${ }^{83}$ a Montgomery-Asberg Depression Rating Scale (MADRS) score $<10$ points, ${ }^{47}$ a MADRS score $<10$ points and a $50 \%$ reduction in symptom score, ${ }^{65}$ a $75 \%$ reduction in HAM-D ${ }_{24}{ }^{72}$ a HAM-D $_{17}$ score $<11.28$ points and a reduction in HAM-D ${ }_{17}$ scores $>7.74$ points ${ }^{68}$ and one study used MADRS not specifying the cut-off for remission. ${ }^{49}$ The RR for lack of remission was 0.78 (95\% CI 0.68 to $0.90 ; \mathrm{p}=0.0008)$ in favour of the intervention using a random-effects analysis. The $\mathrm{I}^{2}$ was $69 \%$ suggesting substantial heterogeneity. The forest plot for the intervention effect on lack of remission is illustrated in online supplementary figure S5.

\section{Missing data}

The scenario in least favour of the intervention was the 'poor' outcome analysis having an effect estimate of RR 0.88 (95\% CI 0.83 to $\left.0.94 ; \mathrm{p}=0.0002 ; \mathrm{I}^{2}=69 \%\right)$. As shown in online supplementary table $\mathrm{S} 1$, the remaining scenarios did not substantially differ from the main analysis.

Heterogeneity and subgroup analysis

$\mathrm{I}^{2}$ was $69 \%$ for the outcome lack of remission suggesting substantial heterogeneity. For this outcome, only two trials $^{2284}$ were considered as trials potentially having less risk of bias than the other trials at high risk of bias. The RR of these two trials was 0.95 (95\% CI 0.74 to $1.23 ; \mathrm{p}=0.78$ ) compared with 0.77 (96\% CI 0.64 to 0.92 ; $\mathrm{p}=0.003$ ) for trials at high risk of bias (test of subgroup difference, $\mathrm{p}=0.19)$. Trials including 52 participants or less in their final analysis had a RR of 0.62 (95\% CI 0.50 to $\left.0.76 ; \mathrm{p}<0.001 ; \mathrm{I}^{2}=45 \%\right)$ compared with 0.95 $\left(95 \%\right.$ CI 0.80 to $\left.1.12 ; \mathrm{p}=0.52 ; \mathrm{I}^{2}=68 \%\right)$ for larger trials (test of subgroup difference, $\mathrm{p}=0.002$ ). Also, trials with a duration of $<10$ weeks had a RR of 0.63 (95\% CI 0.51 to $\left.0.77 ; \mathrm{p}<0.001 ; \mathrm{I}^{2}=40 \%\right)$ compared with $0.93(95 \% \mathrm{CI}$ 0.78 to $1.10 ; \mathrm{p}=0.39 ; \mathrm{I}^{2}=69 \%$ ) for trials of a longer duration (test of subgroup difference, $\mathrm{p}=0.004$ ). As shown in online supplementary table S3, no other trial characteristic significantly explained any of the observed heterogeneity (see online supplementary table S2 for trial characteristics used to explore heterogeneity).

Trial sequential analysis and diversity adjusted required information size

The diversity adjusted required information size for lack of remission was calculated based on our observed diversity of $74 \%$, a proportion in the control group with lack of remission of $66 \%$, an anticipated intervention effect of $15 \%$ relative risk reduction, a risk of type I error of $0.05 \%$ and a power of $90 \%$. As shown in online supplementary figure S6, the cumulative Z-curve just crossed the trial sequential monitoring boundary for benefit. With the 


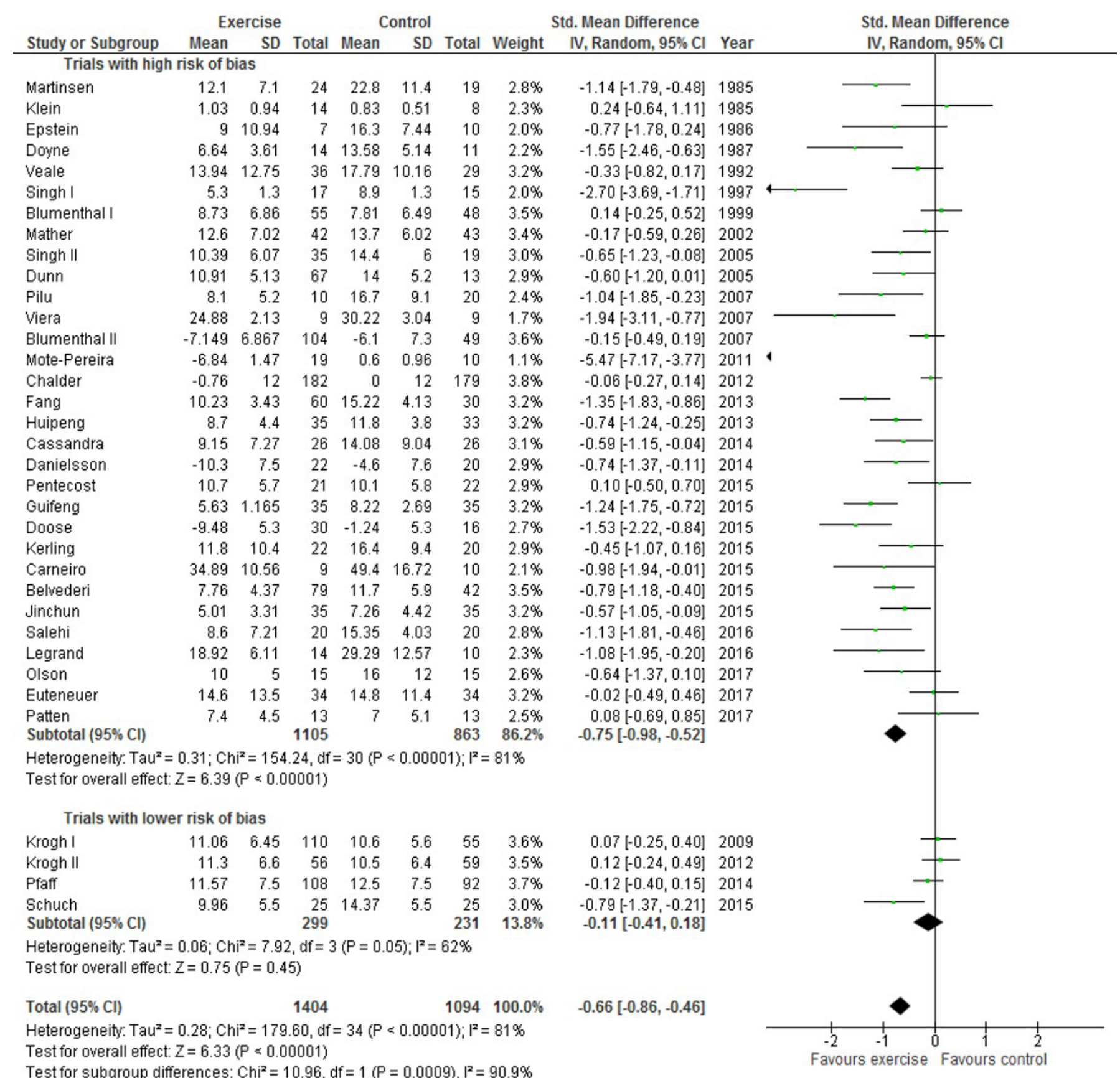


Table 3 Heterogeneity of effect estimates for trials assessing the effect of exercise for patients diagnosed with depression explored by comparing subgroups

\begin{tabular}{|c|c|c|c|}
\hline Subgroups & $\begin{array}{l}\text { Number of } \\
\text { trials (participants) }\end{array}$ & $\begin{array}{l}\text { Random-effects meta-analysis } \\
\text { SMD }\left(95 \% \mathrm{Cl}, \mathrm{p}, \mathrm{I}^{2}\right)\end{array}$ & $\begin{array}{l}\text { Subgroup explains } \\
\text { heterogeneity } \\
\text { p Value }\end{array}$ \\
\hline \multicolumn{4}{|l|}{ Risk of bias } \\
\hline Less than high risk of bias ${ }^{1}$ & $4(530)$ & $-0.11\left(-0.41\right.$ to $\left.0.18 ; p=0.45 ; l^{2}=62 \%\right)$ & $<0.001$ \\
\hline High risk of bias & 31 (1968) & $-0.75\left(-0.98\right.$ to $\left.-0.52 ; p<0.001 ; I^{2}=81 \%\right)$ & \\
\hline \multicolumn{4}{|l|}{ Age } \\
\hline Old (>59years) & $5(492)$ & $-0.77\left(-1.34\right.$ to $\left.-0.19 ; p=0.009 ; 1^{2}=87 \%\right)$ & 0.78 \\
\hline Young (<59years) & 30 (2006) & $-0.68\left(-0.90\right.$ to $\left.-0.45 ; p<0.001 ; I^{2}=83 \%\right)$ & \\
\hline \multicolumn{4}{|l|}{ Exercise context } \\
\hline Group exercise & $26(1785)$ & $-0.75\left(-1.01\right.$ to $\left.-0.50 ; p<0.001 ; l^{2}=83 \%\right)$ & 0.30 \\
\hline Individual exercise & $9(713)$ & $-0.52\left(-0.88\right.$ to $\left.-0.16 ; p=0.005 ; I^{2}=73 \%\right)$ & \\
\hline \multicolumn{4}{|l|}{ Duration } \\
\hline$<10$ weeks & $15(721)$ & $-0.92\left(-1.09\right.$ to $\left.-0.74 ; p<0.001 ; l^{2}=14 \%\right)$ & 0.007 \\
\hline 10 weeks or more & $20(1777)$ & $-0.49\left(-0.75\right.$ to $\left.-0.23 ; p<0.001 ; I^{2}=83 \%\right)$ & \\
\hline \multicolumn{4}{|l|}{ Attention control } \\
\hline Attention control & $10(733)$ & $-0.56\left(-0.98\right.$ to $\left.-0.15 ; p=0.008 ; I^{2}=85 \%\right)$ & 0.91 \\
\hline Waitlist & $2(47)$ & $-0.67\left(-2.48\right.$ to $\left.1.13 ; p=0.47 ; I^{2}=88 \%\right)$ & \\
\hline \multicolumn{4}{|l|}{ Pharmacotherapy } \\
\hline Add-on & $11(734)$ & $-0.92\left(-1.38\right.$ to $\left.-0.46 ; p<0.001 ; I^{2}=86 \%\right)$ & 0.82 \\
\hline No medication & $6(318)$ & $-0.82\left(-1.58\right.$ to $\left.-0.06 ; p=0.03 ; l^{2}=88 \%\right)$ & \\
\hline \multicolumn{4}{|l|}{ Somatic comorbidity } \\
\hline Somatic comorbidity & 0 & $\mathrm{~N} / \mathrm{A}$ & \\
\hline No comorbidity & 35 (2331) & N/A & \\
\hline \multicolumn{4}{|l|}{ Minor depression } \\
\hline Including minor depression & $6(350)$ & $-0.90\left(-1.65\right.$ to $\left.-0.15 ; p=0.02 ;\left.\right|^{2}=86 \%\right)$ & 0.53 \\
\hline No minor depression & $25(2148)$ & $-0.65\left(-0.87\right.$ to $\left.-0.43 ; p<0.001 ; l^{2}=81 \%\right)$ & \\
\hline \multicolumn{4}{|l|}{ Patient setting } \\
\hline Inpatients & $10(549)$ & $-0.88\left(-1.07\right.$ to $\left.-0.70 ; p<0.001 ; I^{2}=6 \%\right)$ & 0.07 \\
\hline Outpatients & $21(1782)$ & $-0.60\left(-0.85\right.$ to $\left.-0.35 ; p<0.001 ; I^{2}=83 \%\right)$ & \\
\hline \multicolumn{4}{|l|}{ Trial size } \\
\hline Trials $n \leq 50$ & $18(578)$ & $-1.11\left(-1.52\right.$ to $\left.-0.72 ; p<0.001 ; I^{2}=78 \%\right)$ & 0.001 \\
\hline Trials $n>50$ & $17(1920)$ & $-0.37\left(-0.57\right.$ to $\left.-0.18 ; p<0.001 ; I^{2}=75 \%\right)$ & \\
\hline \multicolumn{4}{|l|}{ Increase in exercise capacity } \\
\hline $\mathrm{VO}_{2} \max >2.8 \mathrm{~mL} / \mathrm{kg} / \mathrm{min}$ & $5(340)$ & $-0.48\left(-1.08\right.$ to $\left.0.13 ; p=0.12 ; l^{2}=86 \%\right)$ & 0.65 \\
\hline $\mathrm{VO}_{2} \max \leq 2.8 \mathrm{~mL} / \mathrm{kg} / \mathrm{min}$ & $6(661)$ & $-0.32\left(-0.61\right.$ to $\left.0.02 ; p=0.03 ; I^{2}=68 \%\right)$ & \\
\hline
\end{tabular}

Missing data

Missing outcome analysis for 'serious adverse events' varied according to missing data scenario: poor outcome analysis relative risk, 0.92 (95\% CI 0.37 to $\left.2.30 ; \mathrm{p}=0.86 ; \mathrm{I}^{2}=60.0 \%\right)$, good outcome analysis, 2.19 (95\% CI 0.23 to $20.76 ; \mathrm{p}=0.50 ; \mathrm{I}^{2}=0.0 \%$ ), best/worst outcome analysis 0.08 (95\% CI 0.02 to $0.34 ; \mathrm{p}=0.001$; $\mathrm{I}^{2}=5.4 \%$ ), worst/best outcome analysis 19.17 (95\% CI 2.64 to $139.2 ; \mathrm{p}=0.004 ; \mathrm{I}^{2}=0.0 \%$ ).

Trial sequential analysis and Bayes analysis

We decided not to conduct trial sequential analysis or Bayes analysis due to too sparse data.
Publication bias

Only 3/35 trials reported on this outcome and no formal assessment for publication bias was made. However, the lack of reporting in the vast majority of trials suggest risk publication bias.

\section{Secondary outcomes}

The effect of exercise on quality of life

Nine trials randomising 827 participants reported on quality of life, ${ }^{21} 2238405660717685$ observing that participants allocated to exercise did not have significantly better quality of life (SMD 0.40; $95 \%$ CI -0.03 to 0.83 ; 
$\mathrm{p}=0.07)$. The $\mathrm{I}^{2}$ was $88 \%$ showing substantial heterogeneity (see online supplementary figure S8).

\section{Non-serious adverse events}

Non-serious adverse events were reported in only 10 trials. $^{21223956586065676875}$ Five trials reported on musculoskeletal adverse events without conducting formal

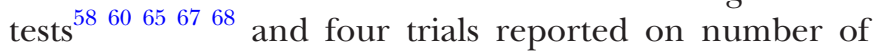
participants with high depression scores postintervention compared with baseline assessment. ${ }^{21} 226568$ The RR for increased severity of depression in patients allocated to exercise postintervention was 0.83 (95\% CI 0.40 to 1.70 ; $\mathrm{p}=0.60 ; \mathrm{I}^{2}=0.0 \%$ ).

\section{The effect of exercise on depression beyond the duration of the} intervention

Assessment of depression beyond the intervention was conducted in seven trials, ${ }^{21} 384052606386$ with a median duration between end of intervention and assessment of depression of 6 months (range 5-23.5 months). The SMD between the intervention group and the control group using a random-effects analysis was -0.10 (95\% CI -0.28 to $\left.0.09 ; \mathrm{p}=0.31 ; \mathrm{I}^{2}=19.5 \%\right)$. The $\mathrm{I}^{2}$ for this estimate was $19.5 \%$ suggesting low heterogeneity (see online supplementary figure S9).

Remission beyond the intervention was assessed in five trials, ${ }^{21} 38-4054$ and the relative risk of lack of remission was $0.95\left(95 \%\right.$ CI 0.82 to $1.11 ; \mathrm{p}=0.53$ ) with an $\mathrm{I}^{2}$ of $0.0 \%$ (see online supplementary figure S10).

\section{GRADE assessments}

The GRADE assessments are presented in table 4, and quality of evidence for both primary and secondary outcomes was very low or low.

\section{Additional analysis}

Four studies reported change in scores from baseline with corresponding SDs, and one study reported mean difference between groups postintervention. Comparing the effect size of these five studies with the remaining did not seem to explain part of the heterogeneity $(p=0.23)$.

\section{DISCUSSION}

Thirty-five clinical trials allocating more than 2498 participants diagnosed with depression according to validated diagnostic instruments were included in the present systematic review. Pooled estimates suggested moderate antidepressant effect assessed both as a continuous outcome and as lack of remission. Due to risk of bias, inconsistency of effect estimates and publication bias, we have, however, very little confidence in these effect estimates. Subgroup analyses exploring reasons for the heterogeneity found that trials potentially having less risk of bias than other trials at high risk of bias had no effect of exercise on depression. Furthermore, duration of intervention and trial size were inversely associated with effect estimates. Exercise did not improve quality of life or depression or remission after the intervention. Serious adverse events or adverse events were reported inconsistently and only by a few trials not permitting firm conclusions regarding these outcomes.

\section{Strengths and limitations}

The strengths of this systematic review are that it is based on the published protocol, a comprehensive search strategy and the inclusion of patient-centred outcomes such as quality of life as well as adverse events. Also, to avoid spurious finding from repeated testing, trial sequential analysis and Bayes analysis were undertaken and these analyses did not suggest that the pooled estimates could be reduced to random errors for effect on depression severity or no remission. Neither trial sequential analysis nor Bayes factor analysis are, however, able to wash of spurious effects induced by bias, fraud or other reasons. ${ }^{26} 2987-89$ Had we restricted the trial sequential analysis to trials of potentially lower risk of bias, the number of trials and participants would be limited and we had seen evidence far from crossing any boundaries for benefit, harms or futility. The conclusions for serious adverse events and adverse events were associated with wide CIs due to lack of data and firm conclusions for these outcomes are presently not available.

The number of trials with adequate allocation concealment was $37 \%$ in the current systematic review compared with only $15.1 \%$ in trials assessing non-drug interventions for depression. ${ }^{90}$ Blinded outcome assessment was performed in $46 \%$ of the included trials compared with $44 \%$ in non-drug antidepressant trials in general. ${ }^{90}$ The incomplete outcome bias domain was adequate in $34 \%$ of our included trials compared with $32.9 \%$ of antidepressant non-drug trials in general. ${ }^{90}$ Compared with non-drug trials assessing interventions for participants with depression, the included exercise trials have more bias domains with low risk of bias. However, all our included trials were at high risk of bias. Two trials had low risk of bias for all bias domains except for blinding of participants and trial personnel, and four trials fulfilled our criteria for trials at potentially less risk of bias than the rest of the trials with at risk of bias. Despite a search strategy including bibliographical databases and trials from China and South America, the vast majority of included trials were conducted in North America and western Europe, which is comparable to the geographical distribution of non-drug trials in general, ${ }^{90}$ limiting the applicability to other geographic regions.

All outcomes for the primary analysis reflect depression severity, however, the different psychometrics may represent different aspects of depression not reflected in the pooled estimate. An in-depth discussion of the included assessment scales is beyond the scope of this review, but in the current systematic review we found no significant differences of effect estimates from trials using HAM-D ${ }_{17}$ compared with trials using other assessment scales (data not shown). 


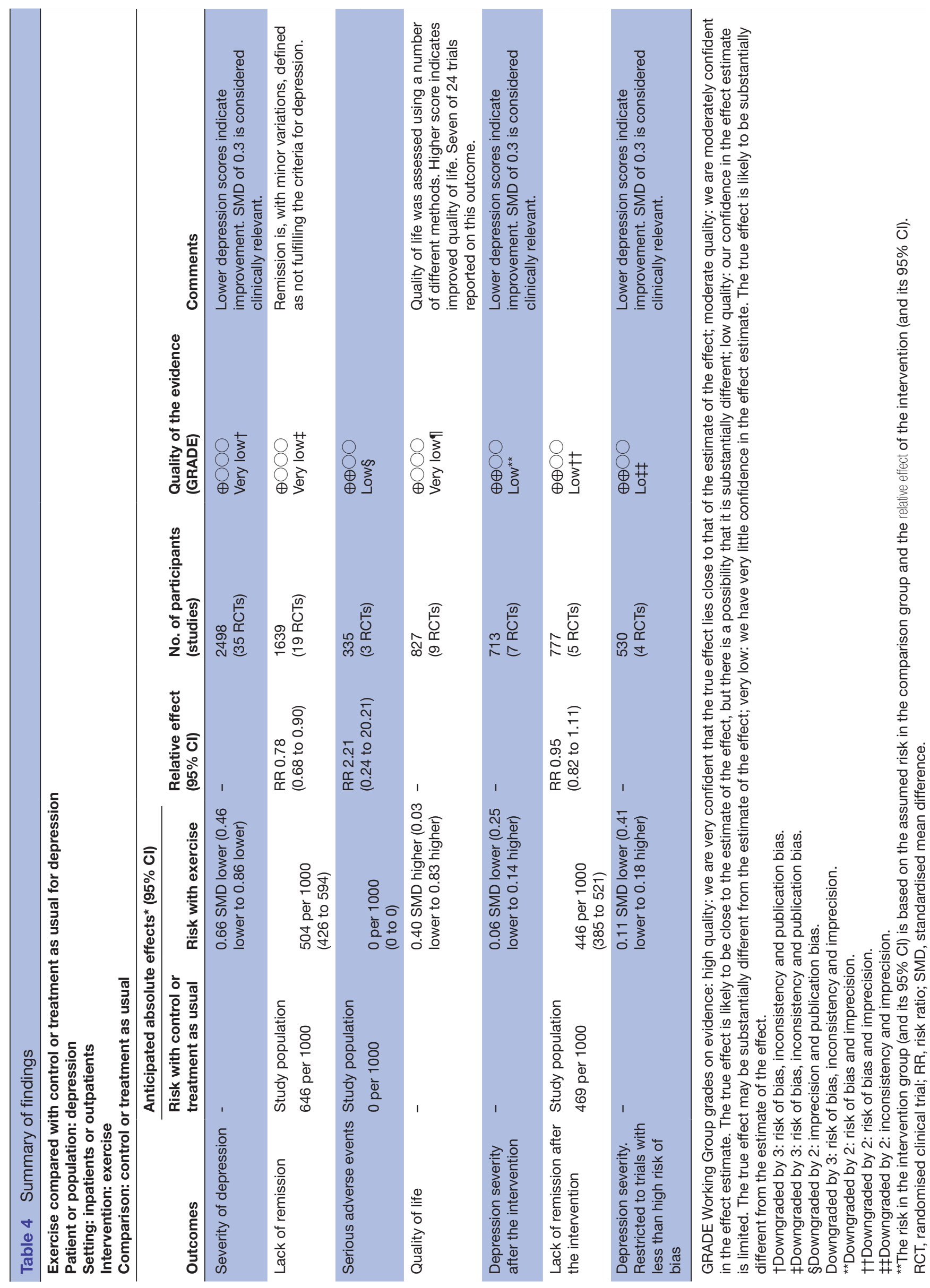

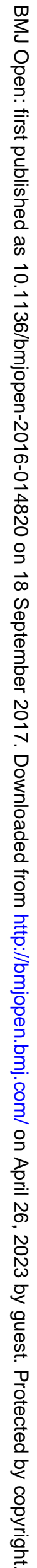




\section{The effect of exercise on depression}

Our present results are similar to the latest Cochrane review by Cooney et $a l,{ }^{24}$ who found a moderate effect of exercise on depressive symptoms (-0.62 SMD) when including all trials and no effect when restricting the analysis to trials with less risk of bias $(-0.18$ SMD). The Cochrane review did find evidence of a small antidepressant effect beyond the intervention, which we could not confirm in our present systematic review. Bridle et $a l^{13}$ included nine trials allocating old ( $>60$ years) participants with depression to exercise interventions versus control interventions. Restricting the analysis to four trials at lower risk of bias they found small-to-moderate effect estimates (SMD -0.34) in favour of exercise. The studies by Cooney $e t a l^{24}$ and Bridle $e t a l^{13}$ both included trials allocating participants with depressive symptoms and not necessarily diagnosed using a validated diagnostic system, potentially explaining the differences in the effect sizes. However, in our present systematic review the estimate for four trials at potential less risk of bias than the remaining trials was -0.11 SMD and in the study by Cooney et al, the effect estimate for eight trials with lower risk of bias was $-0.18 \mathrm{SMD}^{24}$ compared with -0.34 in the study by Bridle et al. ${ }^{13}$ Meta-analysis of randomised clinical trials assessing the effects of exercise for depression consistently finds positive effects, however, when restricting the analysis to trials with less risk of bias the pooled effect sizes becomes very small or negligible. Meta-analysis examining the effect of exercise beyond the intervention also finds no or small effects of exercise. In the process of interpretation of effect estimates in the current research field, it is important to recognise that effect estimates from trials with non-blinded outcome assessment are at high risk of bias as reported by Savovic $e t a l .{ }^{91}$ Sixteen of 35 trials in the current systematic review did not use blinded outcome assessment. In contradiction to the current systematic review, a recent meta-analysis by Schuch $e t \mathrm{al}^{12}$ concluded that 'exercise has a large and significant antidepressant effect in people with depression......... Our data strongly support the claim that exercise is an evidence-based treatment for depression'. This statement was based on a meta-analysis of 25 randomised clinical trials including participants with depression or depressive symptoms to exercise or control conditions and excluding trials using any form of active control group. Surprisingly, the authors found that adjusting for publication bias using the trim and fill procedure, ${ }^{31}$ the estimate increased from an SMD of 0.98 to 1.11. The effect in SMD in included studies ranged from -0.23 to 4.56 representing considerable heterogeneity. ${ }^{12}$ The authors classified four trials as having lower risk of bias using the same criteria as in our systematic review and 21 trials as having high risk of bias. This illustrates some of the challenges in meta-analysis of exercise and depression: the large heterogeneity driven by small studies inflating the effects of random-effects analysis, ${ }^{92}$ the misconception that we can restrict our analysis to statistics and not consider the evident effect of bias. ${ }^{23}$ Compared with our previous review, ${ }^{10}$ we now included 35 trials including 2498 participants versus previously 13 trials and 687 participants. It may seem as a paradox that this large increase in data has not provided us with a similar increase in certainty of conclusions reflected by heterogeneity of trial results as well as our conclusions from the systematic reviews. The increase in available data is, however, primarily provided by small trials at high risk of bias introducing exaggerated effect estimates. In the current systematic review, we included four trials with 530 participants at lower risk of bias compared with three trials with 239 participants in our previous review, reflecting that only a small part of the additional data comes from trials at lower risk of bias. The continuous increase in data associated with high risk of bias will not provide patients, clinicians or policymakers with adequate information and represents an unethical enrolment of trial participants and waste of resources. ${ }^{93-99}$ We therefore recommend that future systematic reviews and meta-analysis a priori should have a primary outcome restricting effect analysis to larger trials with lower risk of bias and that any recommendations regarding exercise interventions for participants with depression should be assessed with the GRADE framework.

The $\mathrm{I}^{2}$ of $81 \%$ and $69 \%$ for the primary outcomes indicate substantial evidence of heterogeneity of intervention effects that is variation in effect estimates beyond chance. Part of this heterogeneity was explained by bias and by trial size: trials at high risk of bias or small trials have very large effect estimates compared with trials potentially at less risk of bias or larger trials. The funnel plots and Egger's test indicate publication bias, however, the association between trial size and effect estimates could suggest that the asymmetry in the funnel plots are due to small study bias rather than publication bias. ${ }^{100}$ It could be argued that both the delivery of exercise as well as the actual increase in fitness are fundamental to the assessment of the antidepressant effects of exercise, and in line with our previous review, we found duration of intervention inversely associated with effect size. ${ }^{11}$ Comparing different exercise intensities, we did find a small effect of high-intensity exercise compared with lower-intensity exercise. However, assessing delivered exercise expressed as increase in maximal oxygen uptake we could not reproduce this finding. Future trials need to pay more attention to the dose of the intervention as well as compliance with intervention. ${ }^{101}$ We suggest using maximal oxygen uptake or one repetition maximum as the gold standard to assess the received exercise. Several studies compare exercise with control interventions rather than waitlist control to reduce the effect of non-specific effects, for example, the DEpression og MOtion (DEMO) trials and the trials by Mather et al. ${ }^{21} 2252$ Also, it could be speculated that the effect of exercise would be harder to detect if participants also received medical treatment in addition. The current systematic review could not confirm that the type of control condition explained heterogeneity. The discussion of control group is important in non-drug trials: choosing a waitlist control group the results potentially 
reflects non-specific effects, choosing an active control group (eg, relaxation exercise) the trial is potentially a comparison between two active treatments. However, in the current systematic review we found no evidence that trials using an attention control group or exercise as add-on to pharmacotherapy had significantly different effect estimates compared with other trials.

Our systematic review did not find indications of a positive effect on quality of life in participants with depression allocated to exercise interventions, which is in concordance with the review by Cooney et al. ${ }^{24}$ Only $3 / 35$ trials reported on serious adverse events, and we found no significant effects of exercise on risk of death or suicide attempt. No indication of increased severity of depression or other adverse events in participants allocated to exercise could be detected. However, data on adverse events were reported sporadically in a minority of trials and currently it is not possible to conclude on the risk of serious adverse events or adverse event from exercise interventions in participants with depression.

\section{CONCLUSIONS}

We have little confidence in the pooled effect estimates, especially because trials with less than high risk of bias produced significantly lower effect estimates, suggesting that exercise interventions only produce small or negligible antidepressant effects, depending on how much of the effect is caused by bias and how much is caused by the intervention. There was no effect of exercise on depression beyond the intervention itself. We found no effect on quality of life. There is currently no evidence in favour of exercise for patients with depression with a view to ameliorate depressive symptoms. Our systematic review did not evaluate possible beneficial effects of exercise on, for example, metabolism or cardiovascular fitness, ${ }^{22} 102$ and it is possible that exercise may have beneficial effects on these factors in patients diagnosed with depression.

\section{Future perspectives}

Despite the large number of published trials, further trials with more robust methodology seem still required to establish progress in this field. Also, additional trials from outside North America and Europe may be required for results to be valid for patients in Asia, Africa and South America. To further elaborate on the current findings, we recommend that future trials must include blinded outcome assessors and outcomes assessing quality of life, metabolic effects and long-term effects beyond the intervention. It is also important that future trials systematically collect and report data on death, suicide events, musculoskeletal injuries and other potential adverse effects in both the intervention group as well as in the control group. Moreover, future trials ought to be designed according to the standard protocol items: recommendations for interventional trials (SPIRIT) guidelines and reported according to the consolidated standards for reporting of trials (CONSORT) guidelines ${ }^{103} 104$ and transparently report deidentified individual participant data enabling individual participant data meta-analyses. ${ }^{105}$

Acknowledgements The authors appreciate the help from Youling He with the Chinese Wanfang bibliographical database and translation of Chinese papers. The authors also thank Janus C Jakobsen for assistance with the calculation of Bayes factor.

Contributors JK conceived the project, collected data, did the statistical analysis, analysed the data, drafted and revised the manuscript. He is guarantor. $\mathrm{CH}$ collected the data, analysed the data and revised the manuscript. HS conceived the project, collected data, analysed the data and revised the manuscript. CG conceived the project, analysed the data and revised the manuscript. MN conceived the project, analysed the data and revised the manuscript.

Competing interests $\mathrm{K}, \mathrm{CG}$, and MN have previously published two trials and a meta-analysis on this topic, which could introduce an academic bias in the current systematic review. We asked new authors ( $\mathrm{HS}$ and $\mathrm{CH}$ ) to be involved in the preparation of the protocol, trial selection and bias assessment. No support from any organisation was received for the submitted work; no financial relationship with any organisations that might have an interest in the submitted work in the previous three years; and apart from the above no other relationship or activities that could appear to have influenced the submitted work.

Provenance and peer review Not commissioned; externally peer reviewed.

Data sharing statement All data used in this study are available in figures and tables. No other data were used.

Open Access This is an Open Access article distributed in accordance with the Creative Commons Attribution Non Commercial (CC BY-NC 4.0) license, which permits others to distribute, remix, adapt, build upon this work non-commercially, and license their derivative works on different terms, provided the original work is properly cited and the use is non-commercial. See: http://creativecommons.org/ licenses/by-nc/4.0/

(c) Article author(s) (or their employer(s) unless otherwise stated in the text of the article) 2017. All rights reserved. No commercial use is permitted unless otherwise expressly granted.

\section{REFERENCES}

1. Lepine JP, Gastpar M, Mendelwicz J, et al. Depression in the community: the first pan-european study DEPRES (Depression Research in European Society). Int Clin Psychopharmocol 1997;12:19-29.

2. Pedersen $\mathrm{CB}$, Mors $\mathrm{O}$, Bertelsen $\mathrm{A}$, et al. A comprehensive nationwide study of the incidence rate and lifetime risk for treated mental disorders. JAMA Psychiatry 2014;71:573-81.

3. Ustün TB, Ayuso-Mateos JL, Chatterji S, et al. Global burden of depressive disorders in the year 2000. Br J Psychiatry 2004;184:386-92.

4. Kirsch I, Deacon BJ, Huedo-Medina TB, et al. Initial severity and antidepressant benefits: a meta-analysis of data submitted to the Food and Drug Administration. PLoS Med 2008;5:e45.

5. Turner EH, Matthews AM, Linardatos E, et al. Selective publication of antidepressant trials and its influence on apparent efficacy. $N$ Engl J Med 2008;358:252-60.

6. Jakobsen JC, Katakam KK, Schou A, et al. Selective serotonin reuptake inhibitors versus placebo in patients with major depressive disorder. A systematic review with meta-analysis and trial sequential analysis. BMC Psychiatry 2017;17:58.

7. Jakobsen JC, Hansen JL, Storebø OJ, et al. The effects of cognitive therapy versus 'no intervention' for major depressive disorder. PLoS One 2011;6:e28299.

8. Jakobsen JC, Hansen JL, Simonsen E, et al. The effect of interpersonal psychotherapy and other psychodynamic therapies versus 'treatment as usual' in patients with major depressive disorder. PLoS One 2011;6:e19044.

9. Jakobsen JC, Hansen JL, Simonsen E, et al. The effect of adding psychodynamic therapy to antidepressants in patients with major depressive disorder. A systematic review of randomized clinical trials with meta-analyses and trial sequential analyses. $J$ Affect Disord 2012;137:4-14.

10. Cipriani A, Furukawa TA, Salanti G, et al. Comparative efficacy and acceptability of 12 new-generation antidepressants: a multipletreatments meta-analysis. Lancet 2009;373:746-58. 
11. Krogh J, Nordentoft M, Sterne JA, et al. The effect of exercise in clinically depressed adults: systematic review and meta-analysis of randomized controlled trials. J Clin Psychiatry 2011;72:529-38.

12. Schuch FB, Vancampfort D, Richards J, et al. Exercise as a treatment for depression: a meta-analysis adjusting for publication Bias. J Psychiatr Res 2016;77:42-51.

13. Bridle C, Spanjers K, Patel S, et al. Effect of exercise on depression severity in older people: systematic review and meta-analysis of randomised controlled trials. $\mathrm{Br} \mathrm{J}$ Psychiatry 2012;201:180-5.

14. Herring MP, Puetz TW, O'Connor PJ, et al. Effect of exercise training on depressive symptoms among patients with a chronic illness: a systematic review and meta-analysis of randomized controlled trials. Arch Intern Med 2012;172:101-11.

15. World Health Organisation. International Statistical Classificationclassification of Diseasesdiseases, 10th Revision (ICD 10. Geneva, Switzerland, 1992.

16. American Psychiatric Association. Diagnostic and statistical manual of mental disorders. 4th ed. Washington DC, 1994.

17. Beck AT, Steer RA, Brown GK. BDI-II manual. 2 ed. New York: Psychological Corporation, 1996.

18. Krogh J, Speyer H, Gluud C, et al. Exercise for patients with major depression: a protocol for a systematic review with meta-analysis and trial sequential analysis. Syst Rev 2015;4:40.

19. Spitzer RL, Endicott J, Williams JB, et al. Research diagnostic criteria. Arch Gen Psychiatry 1979;36:1381-3.

20. ICH-secretariat. International Conference on Harmonisation (ICH) Harmonised Tripartite Guideline (Step 4). Guideline for Good Clinical practice. Geneva, 1996.

21. Krogh J, Saltin B, Gluud C, et al. The DEMO trial: a randomized, parallel-group, observer-blinded clinical trial of strength versus aerobic versus relaxation training for patients with mild to moderate depression. J Clin Psychiatry 2009;70:790-800.

22. Krogh J, Videbech P, Thomsen C, et al. DEMO-II trial. Aerobic exercise versus stretching exercise in patients with major depression-a randomised clinical trial. PLoS One 2012;7:e48316.

23. GSe H. Cochrane Handbook for Systematic Reviews of Interventions Version 5.1.0: The Cochrane Collaboration, 2009. www.cochrane-handbook.org

24. Cooney GM, Dwan K, Greig CA, et al. Exercise for depression. Cochrane Database Syst Rev 2013;9:CD004366.

25. Higgins JP, Thompson SG, Deeks JJ, et al. Measuring inconsistency in meta-analyses. BMJ 2003;327:557-60.

26. Wetterslev J, Thorlund K, Brok J, et al. Trial sequential analysis may establish when firm evidence is reached in cumulative metaanalysis. J Clin Epidemiol 2008;61:64-75.

27. Brok J, Thorlund K, Gluud C, et al. Trial sequential analysis reveals insufficient information size and potentially false positive results in many meta-analyses. J Clin Epidemiol 2008;61:763-9.

28. Thorlund K, Walter SD, Johnston BC, et al. Pooling health-related quality of life outcomes in meta-analysis-a tutorial and review of methods for enhancing interpretability. Res Synth Methods 2011;2:188-203.

29. Jakobsen JC, Wetterslev J, Winkel P, et al. Thresholds for statistical and clinical significance in systematic reviews with meta-analytic methods. BMC Med Res Methodol 2014;14:120.

30. Hollis $\mathrm{S}$, Campbell $\mathrm{F}$. What is meant by intention to treat analysis? survey of published randomised controlled trials. BMJ 1999;319:670-4.

31. Duval S, Tweedie R. Trim and fill: a simple funnel-plot-based method of testing and adjusting for publication bias in metaanalysis. Biometrics 2000;56:455-63.

32. Guyatt GH, Oxman AD, Vist GE, et al. GRADE: an emerging consensus on rating quality of evidence and strength of recommendations. BMJ 2008;336:924-6.

33. Andrews J, Guyatt G, Oxman AD, et al. GRADE guidelines: 14 going from evidence to recommendations: the significance and presentation of recommendations. J Clin Epidemiol 2013;66:719-25.

34. Zheng YP, Lin KM, Zhao JP, et al. Comparative study of diagnostic systems: Chinese classification of mental Disorders-Second Edition versus DSM-III-R. Compr Psychiatry 1994;35:441-9.

35. Babyak M, Blumenthal JA, Herman S, et al. Exercise treatment for major depression: maintenance of therapeutic benefit at 10 months. Psychosom Med 2000;62:633-8.

36. Baxter H, Winder R, Chalder M, et al. Physical activity as a treatment for depression: the TREAD randomised trial protocol. Trials 2010;11:105.

37. Hoffman BM, Blumenthal JA, Babyak MA, et al. Exercise fails to improve neurocognition in depressed middle-aged and older adults. Med Sci Sports Exerc 2008:40:1344-52.
38. Blumenthal JA, Babyak MA, Moore KA, et al. Effects of exercise training on older patients with Major depression. Arch Intern Med 1999;159:2349-56.

39. Blumenthal JA, Babyak MA, Doraiswamy PM, et al. Exercise and pharmacotherapy in the treatment of major depressive disorder. Psychosom Med 2007;69:587-96.

40. Chalder M, Wiles NJ, Campbell J, et al. Facilitated physical activity as a treatment for depressed adults: randomised controlled trial. BMJ 2012;344:e2758.

41. Chalder M, Wiles NJ, Campbell J, et al. A pragmatic randomised controlled trial to evaluate the cost-effectiveness of a physical activity intervention as a treatment for depression: the treating depression with physical activity (TREAD) trial. Health Technol Assess 2012;16:1-iv.

42. Combs K, Smith PJ, Sherwood A, et al. Impact of sleep complaints and depression outcomes among participants in the standard medical intervention and long-term exercise study of exercise and pharmacotherapy for depression. J Nerv Ment Dis 2014;202:167-71

43. Doyne EJ, Ossip-Klein DJ, Bowman ED, et al. Running versus weight lifting in the treatment of depression. J Consult Clin Psychol 1987;55:748-54.

44. Dunn AL, Trivedi MH, Kampert JB, et al. The DOSE study: a clinical trial to examine efficacy and dose response of exercise as treatment for depression. Control Clin Trials 2002;23:584-603.

45. Dunn AL, Trivedi MH, Kampert JB, et al. Exercise treatment for depression: efficacy and dose response. Am J Prev Med 2005;28:1-8.

46. Herman S, Blumenthal JA, Babyak M, et al. Exercise therapy for depression in middle-aged and older adults: predictors of early dropout and treatment failure. Health Psychol 2002;21:553-63.

47. Ho Cwh, Chan SC, Wong JS, et al. Effect of Aerobic Exercise Training on Chinese Population with Mild to Moderate Depression in Hong Kong. Rehabilitation Research and Practice 2014. doi:10.1155/2014/627376

48. Hoffman BM, Babyak MA, Craighead WE, et al. Exercise and pharmacotherapy in patients with Major depression: one-year follow-up of the SMILE study. Psychosom Med 2011;73:127-33

49. Kerling A, Tegtbur U, Gützlaff E, et al. Effects of adjunctive exercise on physiological and psychological parameters in depression: a randomized pilot trial. J Affect Disord 2015;177 1:-6.

50. Khatri P, Blumenthal JA, Babyak MA, et al. Effects of Exercise training on cognitive functioning among depressed older men and women. J Aging Phys Act 2001;9:43-57.

51. Krogh J, Petersen L, Timmermann M, et al. Design paper: the DEMO trial: a randomized, parallel-group, observer-blinded clinical trial of aerobic versus non-aerobic versus relaxation training for patients with light to moderate depression. Contemp Clin Trials 2007;28:79-89.

52. Mather AS, Rodriguez C, Guthrie MF, et al. Effects of exercise on depressive symptoms in older adults with poorly responsive depressive disorder: randomised controlled trial. $\mathrm{Br} \mathrm{J}$ Psychiatry 2002;180:411-5.

53. Mota-Pereira J, Silverio J, Carvalho S, et al. Moderate exercise improves depression parameters in treatment-resistant patients with Major depressive disorder. J Psychiatr Res 2011;45:1005-11.

54. Pfaff JJ, Alfonso H, Newton RU, et al. ACTIVEDEP: a randomised, controlled trial of a home-based exercise intervention to alleviate depression in middle-aged and older adults. $\mathrm{Br} J$ Sports Med 2014:48:226-32.

55. Pilu A, Sorba M, Hardoy MC, et al. Efficacy of physical activity in the adjunctive treatment of major depressive disorders: preliminary results. Clin Pract Epidemiol Ment Health 2007;3:8

56. Schuch FB, Vasconcelos-Moreno MP, Borowsky C, et al. Exercise and severe depression: preliminary results of an add-on study. $J$ Affect Disord 2011;133:615-8.

57. Singh NA, Clements KM, Fiatarone MA. A randomized controlled trial of the effect of exercise on sleep. Sleep 1997;20:95-101.

58. Singh NA, Stavrinos TM, Scarbek Y, et al. A randomized controlled trial of high versus low intensity weight training versus general practitioner care for clinical depression in older adults. J Gerontol $A$ Biol Sci Med Sci 2005;60:768-76.

59. Singh NA, Clements KM, Singh MA. The efficacy of exercise as a long-term antidepressant in elderly subjects: a randomized, controlled trial. J Gerontol A Biol Sci Med Sci 2001;56:M497-M504.

60. Singh NA, Clements KM, Fiatarone MA. A randomized controlled trial of progressive resistance training in depressed elders. $J$ Gerontol A Biol Sci Med Sci 1997:52:M27-M35.

61. Toni G, Belvederi MM, Zanetidou S, et al. Physical exercise for late life Major depression: the SEEDS study. Eur J Prev Cardiol 2014;21:S107. 
62. Veale D, Fk L, Pantelis C, et al. Aerobic exercise in the adjunctive treatment of depression: a randomized controlled trial. J $R$ Soc Med 1992;85:541-4.

63. Vieira JLL, Porcu M, da Rocha PGM. The regular physical exercise's practice as complementary therapy to the treatment of depressed women. J Bras Psiquiatr 2007;56:23-8.

64. Epstein D. Aerobic activity versus group cognitive therapy: an evaluative study of contrasting interventions for the alleviation of clinical depression (dissertation). Reno: University of Nevada, 1986.

65. Danielsson L, Papoulias I, Petersson EL, et al. Exercise or basic body awareness therapy as add-on treatment for major depression: a controlled study. J Affect Disord 2014;168:98-106.

66. Carneiro LS, Fonseca AM, Vieira-Coelho MA, et al. Effects of structured exercise and pharmacotherapy vs. pharmacotherapy for adults with depressive symptoms: A randomized clinical trial. $J$ Psychiatr Res 2015;71:48-55.

67. Legrand FD, Neff EM. Efficacy of exercise as an adjunct treatment for clinically depressed inpatients during the initial stages of antidepressant pharmacotherapy: an open randomized controlled trial. J Affect Disord 2016;191:139-44.

68. Doose M, Ziegenbein M, Hoos O, et al. Self-selected intensity exercise in the treatment of Major depression: a pragmatic RCT. Int J Psychiatry Clin Pract 2015;19:266-75.

69. Salehi I, Hosseini SM, Haghighi M, et al. Electroconvulsive therapy $(E C T)$ and aerobic exercise training (AET) increased plasma BDNF and ameliorated depressive symptoms in patients suffering from Major depressive disorder. J Psychiatr Res 2016;76:1-8.

70. Huipeng $F$, Xiaohui $Z$. Curative effect and observation of exercise on patients with depression. Chin Med Pharm 2013;3:187-8.

71. Jinchun W, Bo Y, Rong X, et al. Effect of exercise therapy in improving social function and quality of life in patients with depression. Nurs J Chin PLA 2015;32:24.

72. Guifeng $\mathrm{S}$. The role of setting - up exercise on rehabilitation in patients with depressive episode. Chin J Health Psychol 2015;23:184-6.

73. Fang $\mathrm{D}, \mathrm{Min} \mathrm{R}, \mathrm{Ai}$-qin $\mathrm{W}$. The effect of aerobic exercise with different intensity and frequency on depressive symptoms. Chin $J$ Behav Med \& Brain Sci 2013;22:830-2.

74. Olson RL, Brush CJ, Ehmann PJ, et al. A randomized trial of aerobic exercise on cognitive control in Major depression. Clin Neurophysiol 2017:128:903-13.

75. Euteneuer F, Dannehl K, Del Rey A, et al. Immunological effects of behavioral activation with exercise in major depression: an exploratory randomized controlled trial. Trans/ Psychiatry 2017;7:e1132.

76. Patten CA, Bronars CA, Vickers Douglas KS, et al. Supervised, vigorous intensity exercise intervention for depressed female smokers: A pilot study. Nicotine Tob Res 2017;19:77-86.

77. Pentecost C, Farrand P, Greaves CJ, et al. Combining behavioural activation with physical activity promotion for adults with depression: findings of a parallel-group pilot randomised controlled trial (BAcPAc). Trials 2015;16:367.

78. Farrand P, Pentecost C, Greaves C, et al. A written self-help intervention for depressed adults comparing behavioural activation combined with physical activity promotion with a self-help intervention based upon behavioural activation alone: study protocol for a parallel group pilot randomised controlled trial (BAcPAc). Trials 2014;15:196.

79. Martinsen EW, Medhus A, Sandvik L. Effects of aerobic exercise on depression: a controlled study. BMJ 1985;291:109.

80. Veale D, Le Fevre K, Pantelis C, et al. Aerobic exercise in the adjunctive treatment of depression: a randomized controlled trial. $J$ $R$ Soc Med 1992;85:541-4.

81. Ossip-Klein DJ, Doyne EJ, Bowman ED, et al. Effects of running or weight lifting on self-concept in clinically depressed women. $J$ Consult Clin Psychol 1989;57:158-61.

82. Blumenthal JA, Babyak MA, Moore KA, et al. Effects of exercise training on older patients with Major depression. Arch Intern Med 1999;159:2349-56.
83. Belvederi Murri M, Amore M, Menchetti M, et al. . Physical exercise for late-life major depression. Br J Psychiatry 2015;207:235-42.

84. Schuch FB, Vasconcelos-Moreno MP, Borowsky C, et al. Exercise and severe Major depression: effect on symptom severity and quality of life at discharge in an inpatient cohort. J Psychiatr Res 2015;61:25-32.

85. Carta MG, Hardoy MC, Pilu A, et al. Improving physical quality of life with group physical activity in the adjunctive treatment of Major depressive disorder. Clin Pract Epidemiol Ment Health 2008;4:1.

86. Klein MH, Greist JH, Gurman AS, et al. A Comparative Outcome Study of Group Psychotherapy vs. Exercise Treatments for Depression. Int J Ment Health 1985;13:148-76.

87. Roberts I, Ker K, Edwards P, et al. The knowledge system underpinning healthcare is not fit for purpose and must change. BMJ 2015;350:h2463.

88. Gluud C, Jakobsen J, Imberger G, et al. Re: The knowledge system underpinning healthcare is not fit for purpose and must change Responses to the opposing viewpoints of Roberts and colleagues and Tovey and colleagues. BMJ 2015;350.

89. Thorlund K, Engstrøm J, Wetterslev J, et al. User manual for Trial Sequential Analysis (TSA). Copenhagen, Denmark: Copenhagen Trial Unit, Centre for Clinical Intervention Research:1-115. https:// www ctu dk/tsa

90. Krogh J, Hjorthøj CR, Jakobsen JC, et al. DEPERROR: Risks of systematic errors in drug and non-drug randomized clinical trials assessing intervention effects in patients with unipolar depression. $J$ Affect Disord 2015;179:121-7.

91. Savović J, Jones HE, Altman DG, et al. Influence of reported study design characteristics on intervention effect estimates from randomized, controlled trials. Ann Intern Med 2012;157:429-38.

92. Poole C, Greenland S. Random-effects meta-analyses are not always conservative. Am J Epidemiol 1999;150:469-75.

93. Ioannidis JP, Greenland S, Hlatky MA, et al. Increasing value and reducing waste in research design, conduct, and analysis. Lancet 2014;383:166-75

94. Chan AW, Song F, Vickers A, et al. Increasing value and reducing waste: addressing inaccessible research. Lancet 2014;383:257-66.

95. Moher D, Glasziou P, Chalmers I, et al. Increasing value and reducing waste in biomedical research: who's listening? Lancet 2016;387:1573-86.

96. Glasziou P, Altman DG, Bossuyt P, et al. Reducing waste from incomplete or unusable reports of biomedical research. Lancet 2014;383:267-76.

97. Chalmers I, Bracken MB, Djulbegovic B, et al. How to increase value and reduce waste when research priorities are set. Lancet 2014;383:156-65.

98. Al-Shahi Salman R, Beller E, Kagan J, et al. Increasing value and reducing waste in biomedical research regulation and management. Lancet 2014;383:176-85.

99. Garattini S, Jakobsen JC, Wetterslev J, et al. Evidence-based clinical practice: overview of threats to the validity of evidence and how to minimise them. Eur J Intern Med 2016;32:13-21.

100. Sterne JA, Sutton AJ, loannidis JP, et al. Recommendations for examining and interpreting funnel plot asymmetry in meta-analyses of randomised controlled trials. BMJ 2011;343:d4002.

101. Slade SC, Dionne CE, Underwood M, et al. Consensus on Exercise Reporting Template (CERT): Explanation and elaboration statement. Br J Sports Med 2016:1428-37.

102. Stubbs B, Rosenbaum S, Vancampfort D, et al. Exercise improves cardiorespiratory fitness in people with depression: a meta-analysis of randomized control trials. J Affect Disord 2016;190:249-53.

103. Schulz KF. CONSORT 2010 statement: updated guidelines for reporting parallel group randomized trials. Ann Intern Med 2010;152:726-32.

104. Chan AW, Tetzlaff JM, Gøtzsche PC, et al. SPIRIT 2013 explanation and elaboration: guidance for protocols of clinical trials. $B M J$ 2013;346:e7586.

105. Skoog M, Saarimäki J, luud C, et al. Transparency and registration in clinical research in the nordic countries: Nordic Trial Alliance, NordForsk, 2015:1-108. 